NBER WORKING PAPER SERIES

\title{
THE EFFECT OF PRIVACY REGULATION ON THE DATA INDUSTRY: EMPIRICAL EVIDENCE FROM GDPR
}

\author{
Guy Aridor \\ Yeon-Koo Che \\ Tobias Salz \\ Working Paper 26900 \\ http://www.nber.org/papers/w26900 \\ NATIONAL BUREAU OF ECONOMIC RESEARCH \\ 1050 Massachusetts Avenue \\ Cambridge, MA 02138 \\ March 2020, Revised June 2022
}

This paper was previously circulated as The Economic Consequences of Data Privacy Regulation: Empirical Evidence from the GDPR.We would like to thank Daron Acemoglu, Francesco Decarolis, Glenn Ellison, Sara Ellison, Avi Goldfarb, and seminar and conference participants at Columbia University, ASSA Meetings, the MaCCI/EPoS Virtual IO Seminar, Columbia Data Science Day, FTC PrivacyCon, Yale Law Big Tech \& Antitrust Conference, the ACM Conference on Economics and Computation, BIRSWorkshop on Statistical Methods for Computational Advertising, Toulouse Economics of Platforms Seminar, and ETH Zurich for helpful comments. We would further like to thank William Nelson for his help. Krista Moody provided outstanding research assistance. All errors are our own. The views expressed herein are those of the authors and do not necessarily reflect the views of the National Bureau of Economic Research.

NBER working papers are circulated for discussion and comment purposes. They have not been peer-reviewed or been subject to the review by the NBER Board of Directors that accompanies official NBER publications.

(C) 2020 by Guy Aridor, Yeon-Koo Che, and Tobias Salz. All rights reserved. Short sections of text, not to exceed two paragraphs, may be quoted without explicit permission provided that full credit, including $\odot$ notice, is given to the source. 
The Effect of Privacy Regulation on the Data Industry: Empirical Evidence from GDPR Guy Aridor, Yeon-Koo Che, and Tobias Salz

NBER Working Paper No. 26900

March 2020, Revised June 2022

JEL No. K24,L0,L5,L81

\section{ABSTRACT}

Utilizing a novel dataset from an online travel intermediary, we study the effects of EU's General Data Protection Regulation (GDPR). The opt-in requirement of GDPR resulted in 12.5\% drop in the intermediary-observed consumers, but the remaining consumers are trackable for a longer period of time. Our findings imply that privacy conscious consumers exert privacy externalities on opt-in consumers, making them more predictable. Consistent with this finding, the average value of the remaining consumers to advertisers has increased, offsetting some of the losses from consumer opt-outs.

Guy Aridor

Northwestern University

2211 Campus Dr

Evanston, IL 60208

guy.aridor@kellogg.northwestern.edu

Yeon-Koo Che

Columbia University

420 W. 118th Street, 1029 IAB

New York, NY 10027

yc2271@columbia.edu
Tobias Salz

Department of Economics, E52-404

MIT

Cambridge, MA 02139

and NBER

tsalz@mit.edu 


\section{Introduction}

Technological advances in the past several decades have led to enormous growth in the scale and precision of consumer data that firms collect. These advances have been followed by progress in machine learning and other data processing technologies that have allowed firms to turn data into successful products and services and earn vast economic returns along the way. ${ }^{1}$ However, at the same time, there has been an increasing number of high profile data breaches and a growing feeling of despondency amongst consumers who lack control over this process. ${ }^{2,3}$ Beyond the immediate economic harm resulting from such data breaches consumers might also value privacy for its own sake. ${ }^{4}$ Against this backdrop, government regulators have proposed and enacted data privacy regulation that empowers consumers to have more control over the data that they generate. The European Union was the first to enact such legislation, the General Data Protection Regulation, which has served as a blueprint for privacy legislation in many other countries and US states. ${ }^{5}$ However, we lack empirical evidence on the effectiveness and broader impact of such regulation. Such evidence is critical not only for guiding the design of upcoming regulation, but also to understand fundamental questions in the economics of privacy.

This paper empirically studies the effects of the EU's General Data Protection Regulation (GDPR), in particular, its requirement that consumers be allowed to make an informed, specific, and unambiguous consent to the processing of their data. ${ }^{6}$ The consent requirement provides a frontline defense of privacy for consumers: by denying consent, a consumer can block a website from collecting personal data and sharing it with third-party affiliates. At the same time, consent denial inhibits firms from tracking consumers across time and across websites, thereby building historical profiles of consumers. Without them, these firms may not be able to learn and predict consumer behavior and target their services and advertising accordingly.

\footnotetext{
${ }^{1}$ Several popular press outlets have gone as far as stating that "data is the new oil" meaning that the world's most valuable resource is now data, not oil (e.g. The Economist - The world's most valuable resource is no longer oil but data. Retrieved on January 9th, 2020).

${ }^{2}$ There have been many but among the most prominent are the Cambridge Analytica and Equifax data breaches. Cambridge Analytica harvested the personal data of millions of people's Facebook profiles without their consent and used it for political advertising purposes. The Equifax data breach exposed the names, dates of birth, and social security numbers of 147 million individuals.

${ }^{3}$ We Hate Data Collection. That Doesn't Mean We Can Stop it. New York Times Privacy Survey. Retrieved on January 3 rd, 2020.

${ }^{4}$ For the different motivations for privacy, see, for instance: Acquisti, Taylor and Wagman (2016). As noted by Lin (2022), consumer privacy preferences contain both an instrumental and non-instrumental component.

${ }^{5}$ Those states and countries include, for instance, Brazil, California, Chile, Colorado, India, Maine, Nevada, New Zealand, Utah, Vermont, and Virginia. While such regulation is not entirely novel, the scope and robustness of previous regulation pales in comparison to that of GDPR. Several states in the United States and countries around the world are debating and implementing their own privacy regulations with similar scope and stipulations as GDPR. For more information on the specifics of the various laws and how they relate to GDPR: Privacy Laws Around The Globe. Retrieved on March 10th, 2020.

${ }^{6}$ The precise legal status of the requirement will be discussed in Section 2.1 .
} 
Our investigation focuses on three broad questions. First, to what extent do consumers exercise the consent right enabled by GDPR? Anecdotal and survey evidence suggests that consumers value their privacy. Yet, it has been argued that consumers may not be willing to act on their privacy concerns even at little cost or inconvenience. ${ }^{7}$ We do not yet have clear empirical answers to this question, and consumers' GDPR opt-out decisions could shed light on their "revealed" value of privacy.

Second, how does GDPR change the composition of consumers observed by firms? Even prior to GDPR, consumers were able to protect their privacy by utilizing browser-based privacy protection. However, utilizing these privacy means does not eliminate their footprints altogether but rather simply generate "spurious" identifiers that are difficult for firms to distinguish from genuine footprints left by consumers who do not adopt them. This process creates noise in the data observed by firms that could make it difficult for them to track consumers and predict their behavior. Under the GDPR regime, however, the same consumers may simply opt out, in which case they do not leave any footprints, and this could in principle make the remaining consumers more easily trackable and identifiable. Moreover, consumers may selectively consent based on how loyal and trusting they are to the websites, which could increase the fraction of more persistently identifiable consumers. This raises an interesting question of externalities created by privacy tools on the other consumers and for the firms. To the best of our knowledge, these forms of privacy externalities not only differ from those recognized in the theoretical literature (Choi, Jeon and Kim, 2019; Acemoglu et al., 2019; Bergemann, Bonatti and Gan, 2019) but more importantly have never been empirically identified.

Third, how does the GDPR privacy protection impact firms that rely crucially on consumer data? Specifically, how does consumer opt-out affect firms' abilities to learn and predict consumer behavior and to provide targeted advertising? And how do advertisers react to such a change? These questions are particularly important for the competitive landscape of the digital economy. While big technology firms such as Google or Facebook enjoy virtually unlimited access to consumer data based on their extraordinary reach and presence, many third-party companies can only access the data shared by first-party affiliates. A concern is that the playing field of these firms, already threatened by the big tech companies, may be further weakened by the increased consent requirement of data regulation. ${ }^{8}$ How such a (relative to the internet giants) smaller

\footnotetext{
${ }^{7} \mathrm{~A}$ prevalent theme in the economics of privacy literature consistently finds a privacy paradox - the apparent inconsistency between individual's strong stated preferences for privacy and their willingness to give away personal information at little cost (Acquisti, Taylor and Wagman, 2016). This directly implies that a natural hypothesis is that consumers may ask legislators for such privacy means but, ultimately, make little use of them.

${ }^{8}$ This concern has been raised in the recent literature (Johnson, Shriver and Goldberg, 2020; Peukert et al., 2022) and the popular press (Wall Street Journal - GDPR Has Been a Boon for Google and Facebook. Retrieved on June 2nd, 2020) which show that GDPR led to an increase in market concentration of web trackers, favoring those from Google and Facebook. This concern is exacerbated by the scope of these companies that allows them to collect data
} 
third-party firm copes with GDPR could provide a valuable clue on how data regulation may influence the competitive playing field of the digital economy.

To answer these questions, we use data provided by an anonymous intermediary that contracts with many of the largest online travel agencies and travel meta-search engines around the world. The dataset is uniquely suited for the current inquiries in several respects. An integral part of the intermediary's business is to predict consumer behavior for the host website. Upon each visit by a consumer at an online travel agency (its first-party affiliate), this firm predicts the likelihood of the consumer buying from the website and places advertisements from alternative travel agencies to consumers it deems unlikely to purchase from the host website.

The data links consumers' behavior across time and across websites using cookies (set by the intermediary)-small files stored attached to a consumer's web browser that allow the intermediary to identify consumers. We observe (in anonymized and aggregated form) the same rich consumer information across the host online travel agencies as the intermediary and link them just as the intermediary can. If a consumer does not consent to data sharing using GDPR opt-out, then his/her cookies cannot be stored, so the consumer is no longer observed by the intermediary. We can directly infer consumer privacy choices from the number of consumer visits as seen by this (third-party) intermediary and the change in composition, necessary to answer the first two questions. We also observe revenues from keyword-based online advertising, and observe the output of a proprietary machine learning algorithm that predicts the purchase likelihood, which will help us to address the third question.

Our empirical design exploits the fact that the intermediary contracts with many different platforms all around the world who were differentially affected by the introduction of GDPR. Furthermore, the machine learning algorithm is trained and deployed separately for each online travel website. This means that changes in data on one website, due to GDPR or other factors, do not impact the performance of the algorithm on other websites. We exploit these features of our data and the geographic reach of GDPR to utilize a difference-in-differences design for several outcome variables across major European countries and other countries where GDPR was not implemented. It is important to clarify that our analysis characterizes the overall impact of the policy. There are documented cases of non-compliance (Commission, 2020b) and GDPR may have changed the salience of privacy around its implementation. Our estimates should therefore be viewed in the context of this partial compliance and increased salience.

We find that GDPR resulted in approximately a $12.5 \%$ reduction in total cookies, which provides evidence that consumers are making use of the increased opt-out capabilities mandated by GDPR. However, we find that the remaining set of consumers who do not opt out are more per-

across many different devices and domains that are not feasible for smaller third-party vendors (Wired - All the Ways Google Tracks You-And How to Stop It. Retrieved on June 2nd, 2020). 
sistently trackable. We define trackability as the fraction of consumers whose identifier a website repeatedly observes in its data over some time period. We find that trackability has increased by $8 \%$ under GDPR.

We explore two plausible mechanisms for the increased trackability. The first is that the individuals who make use of GDPR opt-out are primarily substituting away from other browser-based privacy means, such as cookie blockers, cookie deletion, and private browsing. While the latter generates many "bogus" short-lived consumers (as a new ID is assigned to a consumer, thus making her appear as a new user, each time she visits the site), the former-the GDPR opt-out-simply removes these individuals from the data. The second is that consumers may selectively consent; namely, less frequent users of a website are less trusting of its privacy protection. Due to a combination of these mechanisms, the consumers that remain in the data after the implementation of GDPR are more persistently identifiable.

Given this change in consumer composition, we explore the extent to which this affects advertising revenues. In our setting the revenues that we observe come from keyword-based advertising and, further, when consumers opt out they are no longer exposed to advertisements from the third party intermediary. We find that there is an immediate drop in the total number of advertisements clicked and a corresponding immediate decline in revenue. Over time, though, advertisers on average increase their bids for the remaining consumers, leading to a smaller overall decline in revenue. This indicates that the remaining set of consumers are higher value consumers compared to the pre-GDPR set of consumers. Of the two possible mechanisms for increased trackability, this is consistent with consumers substituting away from obfuscation to opt-out, since the opt-out of obfuscators with short search spells allows for advertisers to better attribute purchases to advertisements than before. This increased attribution ability leads to an increase in perceived overall value of consumers by advertisers. Meanwhile, the finding is less consistent with selective consent in the context of the intermediary, since, according to this mechanism, opt-in consumers are more likely to be loyal to the host website and are therefore less valuable targets for ads of the competing OTAs.

Finally, we study the effect that GDPR had on the intermediary's ability to predict consumer behavior. In particular, we study the performance of the classifier used by the intermediary, which is a crucial element of its business. The classifier provides a prediction of the probability that a consumer will purchase on the website where she is currently searching. We find that the ability of the classifier to separate between purchasers and non-purchasers did not significantly worsen after GDPR. If anything, additional analysis suggests that the intermediaries ability to separate between purchasers and non-purchasers should improve in the long run.

Our results suggest a novel form of externalities that privacy-conscious consumers exert on the rest of economy-including other consumers and the firms and advertisers relying on con- 
sumer data. The combination of selective consent and switching away from less efficient browserbased means of privacy protection to explicit opt-out (enabled by data privacy regulation) could expose the digital footprints of those who choose not to protect their privacy and make them more predictable. These externalities have potentially important implications. First, third-party firms will suffer from loss of consumers who opt out, but this loss will be mitigated by the increased trackability of those consumers who remain. Indeed, our analysis suggests that the mitigating effect could be important; while we find a negative point estimate on overall advertising revenue, this decrease is not statistically significant. Meanwhile, the welfare effect on the remaining consumers depends on how their data is used by the firms. If their data is used to target advertising and services to their needs, as appears to be so far the case, the externality is largely positive and they will also be better off. However, if the data is used to extract their surplus-a possibility in the future-, they could be harmed by the increased trackability. ${ }^{9}$

\section{Related Work}

The protection of consumer privacy and its consequences has been studied by economists, legal scholars, and computer scientists for several decades. We contribute to three strands of literature in the economics of privacy.

Consequences of Data Privacy Regulation: A closely related study that also explores the short run effect of GDPR is Goldberg, Johnson and Shriver (2021). We see these two studies as complementary in terms of the data scenario and findings. Our study utilizes data at a more disaggregate level but is confined to one industry whereas Goldberg, Johnson and Shriver (2021) have a broad cross-section of different websites and are able to investigate to what extent the effect of the GDPR works through a user acquisition channel. Instead, we are able to look in more detail at cookie lifetime and how GDPR has affected advertising revenues of third party firms.

Several other papers have studied the impact of the GDPR in other domains (Jia, Jin and Wagman, 2018, 2020; Zhuo et al., 2021; Utz et al., 2019; Degeling et al., 2018). Peukert et al. (2022); Johnson, Shriver and Goldberg (2020) show that GDPR increased market concentration amongst web technology services. Goldfarb and Tucker (2011); Johnson, Shriver and Du (2020) study the effectiveness of previous data privacy regulations on online advertising. Godinho de Matos and Adjerid (2021) conduct an experiment with a European telecommunications provider to test how consumers respond to the more stringent opt-in requirements that are mandated by GDPR. Finally, Johnson (2013) estimates a structural model of advertising auctions and shows through

\footnotetext{
${ }^{9}$ This could occur through personalized pricing. See, for instance, Dubé and Misra (2019) and Buchholz et al. (2022), for welfare quantifications of such pricing policies.
} 
counterfactual calculations that advertisement revenue drops substantially more under an opt-in rather than an opt-out policy. We complement these papers by utilizing the scope of our setting to tie each of these pieces together and characterize how they interact with each other and are impacted by data privacy regulation.

Information Externalities: An important consequence of a consumer's privacy decision is the informational externality generated by that decision, as information revealed by one consumer can be used to predict the behavior of another consumer. ${ }^{10,11}$ Several recent theoretical studies argue how such externalities can lead to the underpricing of data, and results in socially excessive data collection (Fairfield and Engel, 2015; Choi, Jeon and Kim, 2019; Acemoglu et al., 2019; Bergemann, Bonatti and Gan, 2019; Liang and Madsen, 2019). Braghieri (2019) theoretically studies how privacy choices by consumers can have pecuniary externalities on other consumers by affecting firms' incentives for price discrimination. The current paper identifies a novel form of informational externalities. While the existing research focuses on how a consumer's decision to reveal her private data can predict the behavior of, and thus can inflict externalities on, those who do not reveal their data, we recognize externalities that run in the opposite direction. Namely, we show that the decision by a privacy-concerned consumer may increase the trackability of, and thus exert externalities on, the opt-in consumers who choose to reveal their data. More importantly, to the best of our knowledge, this is the first paper that identifies privacy externalities empirically. ${ }^{12}$

Preferences for Privacy: The broader literature on the economics of privacy, recently surveyed in Acquisti, Taylor and Wagman (2016), has studied the privacy preferences of individuals. One prevalent research strand is understanding the privacy paradox, which is the apparent disparity between stated and revealed preference for privacy. In particular, consumers state a strong preference for privacy, but are willing to give up their personal information for small incentives (Berendt, Günther and Spiekermann, 2005; Norberg, Horne and Horne, 2007; Athey, Catalini and Tucker, 2017). Acquisti, John and Loewenstein (2013) use a field experiment to evaluate individual preferences for privacy and find evidence of context-dependence in how individuals value privacy. Using stated preferences via a survey, Goldfarb and Tucker (2012b) show that consumer's

\footnotetext{
${ }^{10}$ There is also an emerging, broadly related, literature that studies implications of a more data-driven economy (Goldfarb and Tucker, 2012a; Einav and Levin, 2014; Chiou and Tucker, 2017; Kehoe, Larsen and Pastorino, 2018; Aridor et al., 2020; Bajari et al., 2019)

${ }^{11}$ Implicit in the study of the effect on consumer predictability is the notion that privacy is not simply about the revelation of a consumer's information but also the ability of a firm to predict the behavior of a consumer. The idea that privacy has a statistical notion is a common thread in the literature on differential privacy (Dwork, 2011; Dwork and Roth, 2014).

${ }^{12}$ Our explanation for these externalities is consistent with work which shows that the inability to link consumers over time may lead to difficulties in measuring experimental interventions (Coey and Bailey, 2016; Lin and Misra, 2022).
} 
privacy concerns have been increasing over time. Lin (2022) shows via a lab experiment that consumer privacy preferences can be broken down into instrumental and non-instrumental components. Our study contributes to this literature by analyzing consumer privacy choices made in a consequential setting, instead of only looking at stated preferences. We find that a significant fraction of consumers utilize the privacy means provided by GDPR, giving suggestive evidence that consumers do value their privacy in consequential settings and not only say that they do.

The paper is structured as follows. Section 2 overviews the relevant details from European privacy law and consumer tracking technology. Section 3 describes the data and empirical strategy that is used for this study. Section 4 provides evidence on the degree to which consumers make use of the privacy tools provided by GDPR. Sections 5 and 6 analyze the extent to which this affects online advertising revenues and prediction, respectively. Section 7 concludes.

\section{Institutional Details and Conceptual Framework}

In this section we discuss European privacy laws and the relevant details of the General Data Protection Regulation. We will then describe how websites track consumers online and how GDPR can affect such tracking. Furthermore, we will comment on how different mechanisms for opt out may affect the advertising market and the ability of the intermediary to predict consumer purchase behavior.

\subsection{European Data Privacy Regulation}

The GDPR was adopted by the European Parliament in April 2016. Companies were expected to comply with the new regulations by May 25th, 2018. ${ }^{13}$ The law required wide-ranging changes in how firms collect, store, and process consumer data. The fines for non-compliance with the rules are large - the maximum is set at $€ 20$ million, or $4 \%$ of total global annual sales for the preceding financial year - giving strong incentives for firms to comply with the regulation, which led them to spend millions of dollars to do so. ${ }^{14}$

We focus on the consumer-facing aspect of the regulation, namely the conditions under which a firm can collect consumer data. Regulators have clarified that the most appropriate legal basis for data collection, especially for the type of data collected by the intermediary, is individual

\footnotetext{
${ }^{13}$ GDPR was intended to overhaul and replace the Data Protection Directive which was enacted in 1995. GDPR further complements the other major European Privacy Regulation, The Privacy and Electronic Communications Directive, also known as the "Cookie Law". Relative to this law, GDPR strengthened the territorial scope to include data generated by EU consumers, no matter the location of the firm processing the data, and strengthened the degree of firm transparency and stipulations on consumer consent.

${ }^{14}$ Pulse Survey - GDPR budgets top $\$ 10$ million for $40 \%$ of surveyed companies. Retrieved on December 15 th, 2019.
} 


\section{Figure 1: Example Consent Notifications}

(a) Post-GDPR consent dialog

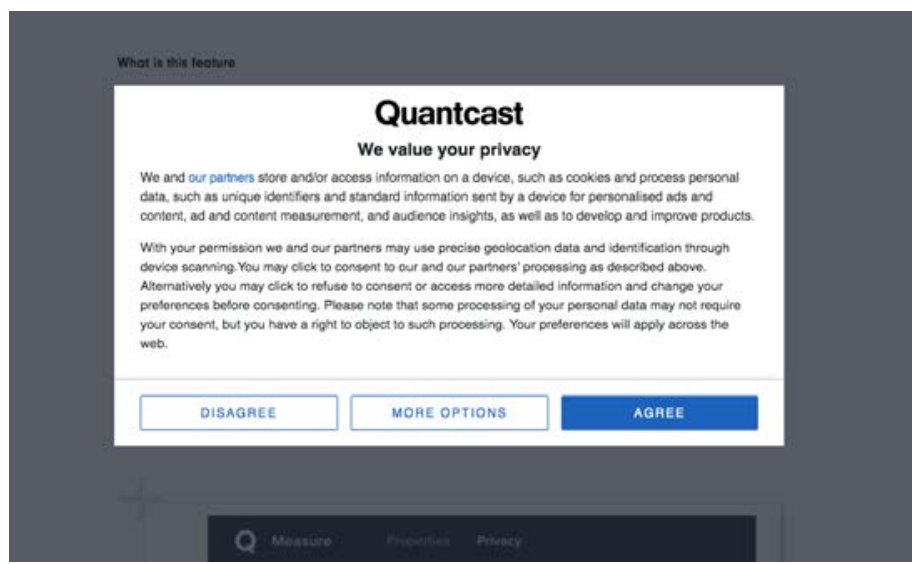

(b) Standard opt-out on US websites

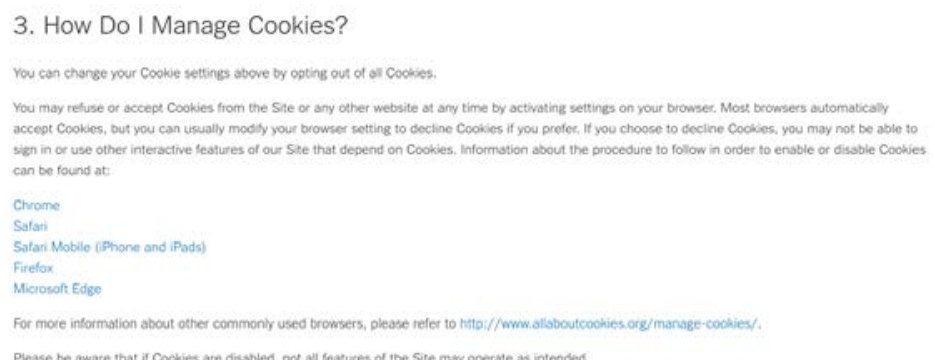

Notes: The top panel shows a standard GDPR opt in consent dialog provided by Quantcast. The dialog is explicit about the data that the website collects and requires the consumer to opt into all non-essential data collection. The bottom panel shows an "opt out" dialog for a website in the US that is not required to be GDPR compliant. The website directs consumers to manage their browser cookies and does not have any direct options for the consumer to opt out of data collection.

consent (Commission, 2020a). From Recital 32 of the regulation, firms need informed, specific, and unambiguous consent from consumers in order to process their personal data, which requires consumers to explicitly opt into data collection:

Consent should be given by a clear affirmative act establishing a freely given, specific, informed and unambiguous indication of the data subject's agreement to the processing of personal data relating to him or her, such as by a written statement, including by electronic means, or an oral statement. This could include ticking a box when visiting an internet website, choosing technical settings for information society services or another statement or conduct which clearly indicates in this context the data subject's acceptance of the proposed processing of his or her personal data. Silence, pre-ticked boxes or inactivity should not therefore constitute consent. 
Panel (a) of Figure 1 shows an example of a commonly used post-GDPR cookie policy from Quantcast and panel (b) of Figure 1 shows a cookie policy of a firm in the United States. The former highlights the specifications of the law, specifying what type of cookies are stored for what purposes and giving consumers the opportunity to opt out from them. By contrast, the latter has no explicit option for the consumers to opt out of data collection. Instead, it directs consumers to use browser-based privacy means, which allows them to control the website's cookies. The intermediary that we partner with is considered a third-party affiliate and, for the typical consent notices, is never explicitly mentioned by name. Thus, it is likely the identity of the OTA, rather than the intermediary itself, is more salient for consumer's opt-in choice in our setting.

It is important to note that limited enforcement of the GDPR paired with ambiguity about which types of consent notices were appropriate have led to observed instances of non-compliance with the regulation and wide heterogeneity in implemented consent notices (Commission, 2020b). This implies that our estimates measure the overall impact of the policy on the outcome variables of interest and not the impact of particular types of consent notices.

\subsection{Potential Effects on Consumers and Firms}

GDPR enabled consumers to opt out of data-sharing. While consumers may opt-out for various reasons, we focus on two plausible ones here. We then discuss their implications for the patterns of observed consumer behavior and their consequences for firms and advertisers. Throughout, these two mechanisms will be used to interpret our empirical findings.

Selective Consent. The first is selective consent, where consumers only consent to data processing by websites that they trust and thus frequently use. According to this mechanism, infrequent users of a website are more likely to opt out of data sharing than frequent users. If this is the primary mechanism for opting out, then one direct consequence is that privacy regulation may favor bigger or more reputed firms. There is a connection of this mechanism to the theoretical predictions in Campbell, Goldfarb and Tucker (2015), who argue that consent-based data collection practices would allow larger firms to collect more data than smaller firms since they offer a wider scope of services. As a result, consumers may visit the websites more and share more data with it. In the long run, this would imply that consent for data collection may serve as a barrier to entry.

This is illustrated in Figure 2. The figure shows the data generated by three consumers. "Full Visibility" shows a hypothetical scenario where each of the three consumers is fully identifiable. The right panel under GDPR shows that the infrequent consumer - consumer 3 - opts out and is no longer observed by the intermediary. From the intermediary's perspective, the remaining consumers will be seen to be more persistently identifiable. 
Figure 2: Illustration of Effects of Selective Consent on Data Observed

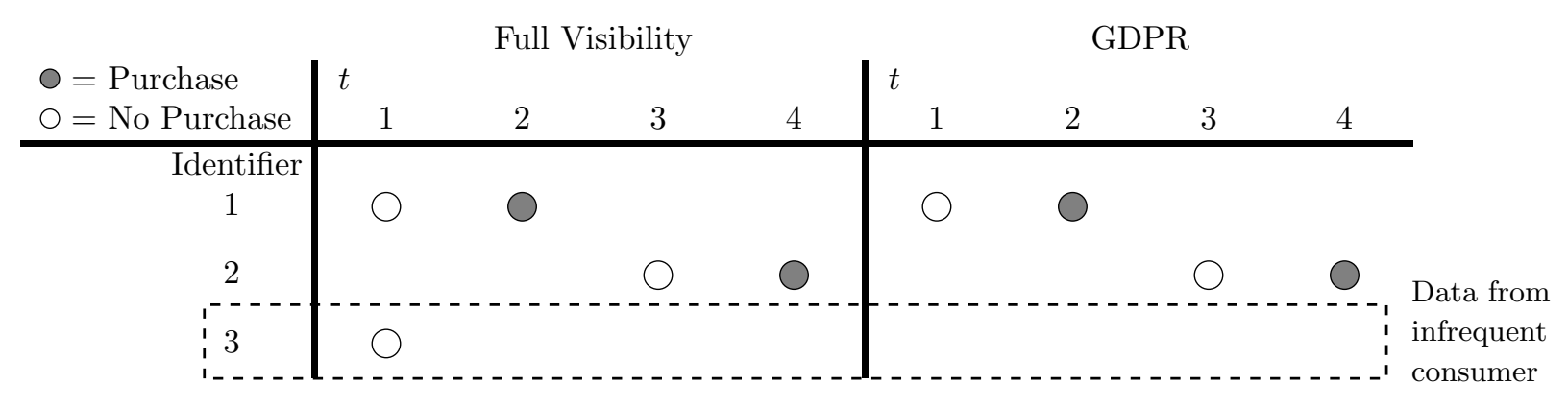

Notes: The leftmost column displays the identifier observed by the intermediary. The left panel represents the scenario where the behavior of each consumer is fully observable. The right panel shows how, under GDPR, the data of the infrequent consumer 3 is not directly sent to the intermediary.

The figure also illustrates how selective consent can impact the intermediary's ability to predict whether a consumer will purchase from the given website. Consider the intermediary's prediction problem for consumer 2 in the full visibility baseline. At $t=3$ its "training data" consists of a mixture of an "infrequent" consumer - consumer 3 - and a "frequent" consumer - consumer 1 - whose data in their first observed search is identical. Thus, the ability of the intermediary to predict whether consumer 2 will ever purchase a flight is more difficult since her search history pools with both types, despite being a "purchaser" type. However, under the GDPR regime, the infrequent consumers opt out of the data, which makes the prediction problem of the intermediary for consumer 2 easier. The key aspect that drives the information externality of consumer 3 is dynamic in nature - if early in the search process the "infrequent" types pool together with the "frequent" types, then GDPR opt out makes it easier for the intermediary to separate the two.

Finally, selective consent would have consequences for the advertising market in our setting. Recall that the intermediary targets the consumers that are deemed less loyal to the host website and thus are more likely to respond to competing OTA's ads. If the opt-out consumers are the infrequent users of the host websites, as suggested by the theory, then the remaining consumers are likely to be those loyal to the host websites and thus unlikely to respond to the competing OTAs' ads. This means that the average value of consumers is likely to decrease after GDPR. Moreover, consumers according to this mechanism only share data with their preferred OTA making it harder to win them over with advertisements for comparison shopping, which is the main kind of advertisement of the intermediary. Hence, we would expect advertisers to spend less on advertisements for comparison shopping and prices to decrease. 
Substitution of the means for protecting privacy. The GDPR opt-out presents consumers with a powerful way of protecting their privacy. For privacy-concerned consumers, this means that they can substitute from other less effective privacy means to simple opt-out. Prior to GDPR, such consumers were likely to employ a variety of methods to protect themselves against consumer tracking by websites. We will denote this type of substitution privacy means substitution.

One prominent method that websites, including our intermediary, use to track consumers is through web cookies. ${ }^{15}$ Cookies are small text files that are placed on consumer's computers or mobile phones. The attachment of a cookie gives websites, in principle, a persistent identifier. As long as the same cookie persists, they can attribute different sessions to the same consumer and, as a result, track them across time and different websites. Privacy-conscious consumers can use various privacy means to control the degree of persistence of this identifier. The primary means available to them are browser-based tools, such as manual deletion of cookies, "private browsing" mode, ${ }^{16}$ or advertising/cookie blockers,${ }^{17}$ all of which we will call obfuscation throughout. One important detail to note is how advertising/cookie blockers work in this context. According to our discussions with employees of the intermediary, these services continually regenerate the identifier utilized by the intermediary while still allowing consumers to see the advertisements. Thus, these consumers leave a distinct mark in the data as "single searchers" who only have one observation associated with their identifier but the data that is generated by them on the website is still sent and stored. Hence, a consumer's data will be attributed to different identifiers. ${ }^{18}$

The stipulations of GDPR, properly implemented and utilized by consumers, arguably provide a stronger protection than the aforementioned means since they block all non-essential information from being sent to the third-party website. ${ }^{19}$ In our context, this means that by simply opting

\footnotetext{
${ }^{15}$ Common alternatives are other forms of storage in the browser as well as device fingerprinting, which use Internet Protocol (IP) addresses combined with device specific information to identify individuals. However, these are less commonly utilized and importantly not utilized by the intermediary.

${ }^{16}$ Private browsing modes create "sandbox" browser environments where cookies are only set and used for the duration of the private browsing session. As a result, the website cannot link together data from the same consumer both before and after the private browsing section.

${ }^{17}$ There also exist industry opt-out services, such as the Ad Choices program, but these are relatively hard to use and have low take up (Johnson, Shriver and Du, 2020). Survey-based evidence informs us that the most utilized manual privacy means by consumers is cookie deletion (Boerman, Kruikemeier and Zuiderveen Borgesius, 2018). Furthermore, there is wide adoption of advertising and cookie blockers (see e.g. Statista - Ad Block Users WorldWide. Retrieved on April 4th, 2022 - which suggests that there are 732.32 million users of ad blockers worldwide in 2018.)

${ }^{18}$ One possible instrumental reason why consumers in this setting would engage in privacy-preserving behavior is that there is a belief, erroneous or not, that cookies are used to implement personalized pricing (see e.g. Business Insider - Clear Cookies When Searching for Flights. Retrieved on April 4th, 2022).

${ }^{19}$ It is important to note that GDPR does not prevent "essential" information from being sent to a website. For instance, the ability to store consumer session cookies that allows them to provide a consistent consumer experience for the consumer may be considered "essential" information. The intermediary that we partner with, however, is a third-party service that provides complementary services to the primary functioning of the websites and so is not an "essential" service on any website where we observe data. As a result, any usage of GDPR opt-out shuts out data from being sent to the intermediary.
} 
out consumers can keep their data from being sent to the intermediary since it provides a nonessential, third-party service. In principle, consumers may still prefer other means to protect their privacy for fear that firms otherwise disadvantage them. However, the GDPR does not allow firms to discriminate against consumers who do not consent to data sharing, making opt-out more attractive all else equal. ${ }^{20}$ Hence, it is plausible that privacy-conscious consumers would substitute from obfuscation to opt-out.

Such substitution will have a distinct effect on the data observed by the intermediary. Before GDPR, reliance on obfuscation by a consumer will mean that her data would still be sent to the intermediary but with many "bogus" identifiers associated with each visit she makes. After GDPR, if such a consumer simply opts out, as postulated by the current mechanism, no data from that consumer is sent to the intermediary. This is the important distinction for our purpose. Browserbased privacy means lead to many artificially short consumer histories that still enter the data, whereas GDPR opt-out removes the data completely. ${ }^{21}$

Figure 3: Illustration of Effects of Obfuscation on Data Observed

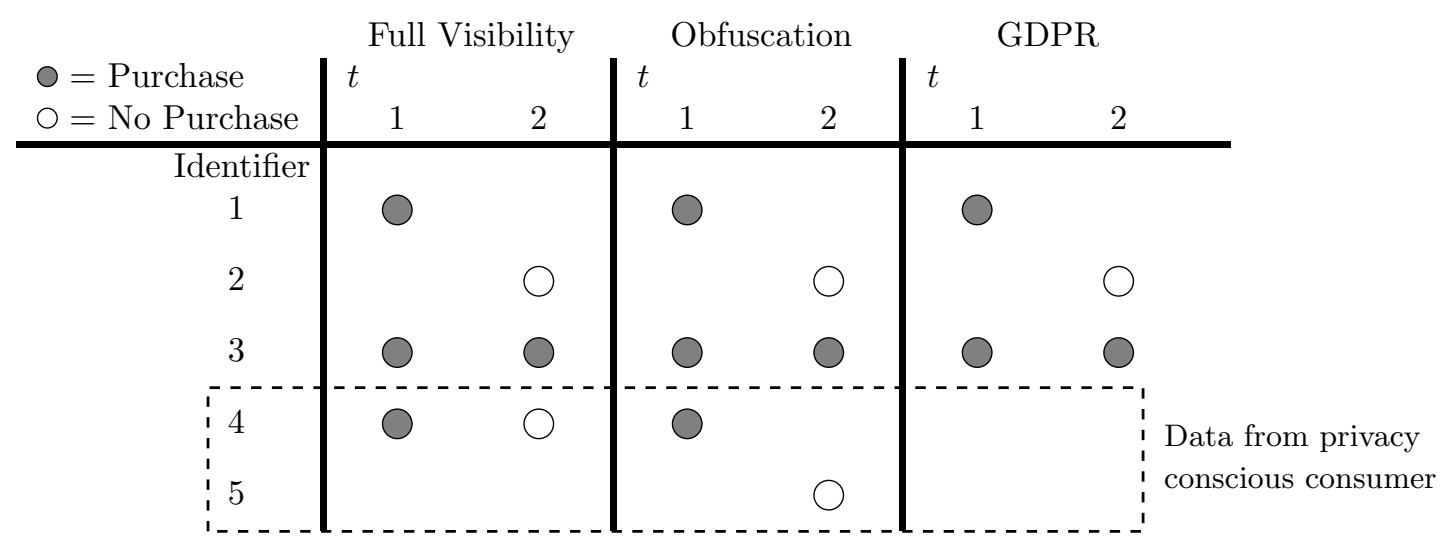

Notes: The leftmost column displays the identifier observed by the intermediary. The left panel represents the scenario where the behavior of each consumer is fully observable. The middle panel shows how, before GDPR, the privacy conscious consumer 4 has her identifier partitioned into two separate identifiers from the perspective of the intermediary. The right panel shows how, under GDPR, the data of the privacy conscious consumer, is not directly sent to the intermediary.

This is illustrated in Figure 3. The figure shows the data generated by four different consumers.

\footnotetext{
${ }^{20}$ In that respect it is more stringent than, for instance, the CCPA that allows firms to set explicit incentives for data sharing. See Cal. Civ. Code $§ 1798.125$ for the CCPA's stipulation and Baker Law - GDPR vs. CCPA Chart (Retrieved on April 6th, 2022) for a comparison of the GDPR and the CCPA.

${ }^{21}$ It's important to point out that consumers can still make use of both privacy means and do not necessarily need to substitute from exclusively using browser-based privacy means towards exclusively using GDPR-provided privacy means. However, from the perspective of the intermediary and websites in general, once a consumer utilizes GDPR opt-out then, since they no longer see any data from this consumer, the browser-based privacy means become irrelevant. As a result, from their perspective, it appears as a direct substitution.
} 
"Full Visibility Baseline" shows a hypothetical scenario where each of the four consumers is fully identifiable. They generate spells of browsing sessions where each dot corresponds to one session and the color of the dot indicates whether or not the consumer purchased a good on the website as a result of that search. Suppose that only consumer 4 is privacy-conscious. Before GDPR, consumer 4 can protect her privacy by deleting her cookies and regenerating her identifier. This is illustrated in the second panel ("Obfuscation") of the figure where the two sessions for this consumer are associated with two separate identifiers from the perspective of the intermediary. However, the third panel shows that, when GDPR opt-out is available, this consumer opts out and her data completely disappears.

The figure also illustrates how the different data scenarios impact the intermediary's ability to predict consumer behavior, and in particular, how a consumer's choice of privacy means may affect that ability. The four consumers have distinct histories, and these differences may signal different future behavior for them. For example, consumer 4 may be less likely than consumer 1 to purchase from the website next time she visits the website. Under Full Visibility, the prediction machine will correctly recognize this distinction and assign a different prediction score to consumer 4 than to consumer 1 . Suppose, however, in the pre-GDPR regime, consumer 4 deletes her cookies and gets partitioned into two separate identifiers, 4 and 5 . This behavior confounds the intermediary's ability to predict not only 4's behavior but also 1 and 2's: consumer 1 is now indistinguishable from consumer 4 and consumer 2 is indistinguishable from consumer 5 (the same person as consumer 4) from the intermediary's view point. For instance, the intermediary will assign a lower than accurate purchase odds to consumer 1, influenced by the fact that consumer 4 with the same history simply disappears after the visit at $t=1$. Note that this problem exists even when the intermediary's prediction machine eventually "learns" about the presence of obfuscators, since it cannot tell who obfuscates and who does not. Under GDPR, on the other hand, consumer 4's data is not observed at all. While this leads to a loss in the amount of data, consumer 4 no longer confounds the prediction for consumer 1 and 2's behavior. ${ }^{22}$

This last point has an implication for the advertisements mediated by the intermediary. As noted earlier, the intermediary targets advertisements to consumers with short spells of cookie histories at the host website, which importantly includes the obfuscators. Prior to GDPR, the obfuscators, with new cookies artificially generated for every visit including one that lead to a purchase, are not easily linked across ad clicks and purchases. In other words, prior to GDPR, a significant presence of the obfuscators makes it difficult for advertisers to "attribute" purchases to ad clicks. Hence, their presence undermines the value of ad clicks as perceived by advertis-

\footnotetext{
${ }^{22}$ Importantly, the pre-GDPR intermediary cannot simply replicate the same dataset as post-GDPR since the obfuscators' identities are latent to the intermediary, so their data cannot be surgically cleaned away; for instance, eliminating single-search data will eliminate not only 4 but also 1 and 2 from the data.
} 
ers. ${ }^{23}$ Suppose, as postulated by the mechanism, a significant number of obfuscators opt out after GDPR. Then, the number of consumers with short spells of cookie histories will decrease, so the number of consumers that the intermediary targets for advertising will fall. However, the remaining consumers targeted by the intermediary-namely the opt-in consumers with short spells of cookie histories-are more likely to be linked across their web visits, so their purchases are more easily attributed to ad clicks. This will increase the average value of consumers (targeted by the intermediary) to the advertisers and should increase prices.

\section{Data and Empirical Strategy}

We obtained access to a new and comprehensive dataset from an anonymous intermediary that records the entirety of consumer search queries and purchases across most major online travel agencies (OTAs) in the United States and Europe as well as most prominent travel meta-search engines. We observe consumer searches, online advertising, and the intermediary's prediction of consumer behavior. Our primary analysis utilizes data from this intermediary ranging from April to July 2018.

\subsection{Data Description}

The disaggregated data contains each search query and purchase made on these platforms as well as the associated advertising auction for each query. In a single search query the data contains: the identifier of the consumer, the time of the query, the details of the query (i.e. travel information), an identifier for the platform, the browser, the operating system, and the estimated probability of purchase on the website according to the predictive machine learning algorithm employed by the intermediary. For a subset of the websites, we observe purchase information containing the consumer identifier and time of purchase.

Each query can trigger an advertising auction. In that case, the data contains: the number of bidders in the auction, the values of the winning bids, and an identifier for the winning bidders. Furthermore, if a consumer clicks on the resulting advertisement, the click itself and the resulting transfer between the advertiser and the intermediary are recorded.

\footnotetext{
${ }^{23}$ To illustrate, suppose that there are five consumers who click on an advertisement. Suppose one of them (from here on consumer $A$ ) makes use of cookie blockers but ends up purchasing and, from the remaining four, suppose two of them end up purchasing. Thus, regardless of the behavior of consumer $A$, the advertiser's estimated conversion rate is 0.4 as opposed to 0.6 -a correct rate including $A$. Suppose, instead, that GDPR opt-out is available and consumer $A$ is removed from the sample of the advertiser and therefore never clicks on an advertisement. The advertiser's estimated conversion rate is 0.5 now, as opposed to 0.4 and so the perceived value of consumers weakly increases regardless of consumer $A$ 's true behavior. More generally, dropping individuals similar to consumer $A$ from the observed sample can only weakly increase the advertiser's perceived value.
} 
Overall, we observe, in aggregated form, data on the searches, advertisements, and purchases that occur on each of the online travel agencies and travel meta-search engines that the intermediary contracts with. Our analysis utilizes an aggregation of this dataset by week, operating system, web browser, website identifier, product type, and country. ${ }^{24}$ The data was aggregated on a weekly level to remove unimportant day-of-the-week fluctuations. Furthermore, the GDPR compliance date was May 25th, 2018, which was on a Friday and, as a result, our data was aggregated on a Friday-to-Friday level. Note that the GDPR compliance date corresponds to the beginning of the $22 \mathrm{nd}$ week in the year according to our labeling. ${ }^{25}$

\subsection{Empirical Strategy}

To understand the causal effect of GDPR we rely on a difference-in-differences design that exploits the geographic reach of the EU GDPR regulation. The regulation stipulates that websites that transact with EU consumers were able to comply with the regulation by asking consumers to consent to data sharing, while this was not necessary for non-EU consumers. Even though many online travel companies transact with consumers in several countries around the world this specification works well in our setting since it is common for online travel websites to have separate, country-specific versions of their websites and only the websites intended for EU countries are made GDPR compliant.

Our analysis captures the overall effect of the policy and not the specific effect of consent implementation. Thus, the treatment date of the policy corresponds to the GDPR compliance date, which was May 25th, 2018 (or the beginning of week 22). Our treatment group consists of nearly the universe of travel websites in major EU countries (at the time): Italy, the United Kingdom, France, Germany, and Spain. Our control group consists of nearly the universe of travel platforms in the United States, Canada, and Russia. These countries were chosen as controls since EU laws do not directly apply to them, but their seasonal travel patterns are similar to those in the EU countries as a result of similar weather and vacation patterns in the time period of interest.

Our primary regression specification is the following for the outcome variables of interest where $c$ denotes country, $j$ denotes the website, $o$ denotes operating system, $b$ denotes web browser, $p$ denotes product type (hotels or flights), and $t$ denotes the week in the year:

$$
y_{t, c, j, o, b, p}=\alpha_{t}+\delta_{j, c}+\gamma_{o}+\zeta_{b}+\omega_{p}+\beta \cdot\left(E U_{j} \times \text { after }_{t}\right)+\epsilon_{t, c, j, o, b, p}
$$

\footnotetext{
${ }^{24}$ We drop from this aggregation observations which are labeled as coming from bots.

${ }^{25}$ Note that we further enforce a balanced panel by dropping any observation that has zero logged searches in any period during our sample period. We do this in order to ensure that our estimates are not biased from entry / exit of websites into our data during the sample period. According to discussions with our data provider, this entry and exit is usually a result of varying contractual relations between the intermediary and the websites and so is largely orthogonal to our variables of interest.
} 


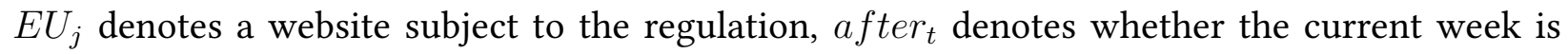
after the GDPR compliance date (i.e. week 22 or later), $\alpha_{t}$ denotes time fixed effects, $\delta_{j, c}$ denotes country-specific website fixed effects, $\omega_{p}$ denotes product type fixed effects, $\gamma_{o}$ denotes operating system fixed effects, and $\zeta_{b}$ denotes browser fixed effects. Our standard errors are clustered at the website-country level. ${ }^{26}$

In order to validate parallel trends and to understand the persistence of the treatment effect, we further utilize a regression specification that captures the potentially time-varying nature of the treatment:

$$
y_{t, c, j, o, b, p}=\alpha_{t}+\delta_{j, c}+\gamma_{o}+\zeta_{b}+\omega_{p}+\sum_{k=\underline{T}}^{\bar{T}} \cdot \beta_{k} E U_{j}+\epsilon_{t, c, j, o, b, p}
$$

The variable definitions are the same as before and we similarly cluster our standard errors at the website-country level.

We run our regressions over the time period between weeks 16 and 29 of 2018, which is between April 13th and July 20th. The GDPR compliance date aligns with the beginning of week 22. Furthermore, week 20 is consistently the baseline week in our regressions since there are some firms that began to implement GDPR near the end of week 21 and so week 20 is the last week where there should be no direct impact from GDPR as a result of website implementation. ${ }^{27,28}$

Our empirical strategy centers around the official GDPR implementation date. However, each website had to individually implement the changes stipulated by GDPR and there is evidence that there was considerable heterogeneity in compliance among firms. Furthermore, even within the subset of firms that complied with the regulation, the degree to which consumers responded varied considerably based on the nature of implementation (Utz et al., 2019). As a result, we would want to include information on the timing and degree of implementation across the various websites in our sample. However, due to technical limitations, we cannot directly observe the timing and degree of GDPR implementation during the time period we study. ${ }^{29}$ Thus, any effects

\footnotetext{
${ }^{26}$ We cluster at the website-country level because of differences in privacy concerns across countries (Prince and Wallsten, 2020) and differences in consent implementations across websites within jurisdiction (Utz et al., 2019).

${ }^{27}$ Our dataset ends on July 31st, 2018, which is a Tuesday, and an important measure that we want to track is the amount of consumer persistence on a weekly level, which looks at the fraction of observed cookies that remain observable in the data after some number of weeks. Since this measure requires a complete week of data to compute properly, we drop the incomplete week at the end of July as well as the full last week in July so that we can have consistency between the regressions on aggregate consumer response and those on consumer persistence.

${ }^{28}$ Our analysis ends at the end of July since this was the time period over which we were able to obtain data from our data provider.

${ }^{29}$ We attempted to utilize tools such as the Wayback Machine, which takes snapshots of websites across the entire Internet frequently. However, the coverage of relevant websites on the Wayback Machine is spotty and, given that many of the consent dialogs for GDPR consent are dynamically generated, are not always picked up by the snapshot taken of the website. We were able to verify that several websites in our sample implemented GDPR consent guidelines around the time of the policy and that several websites in our sample did not, though there are a
} 
that we observe with our empirical specification are a combination of the explicit consequences of implementing the stipulations of GDPR for the subset of websites that implemented it and any changes in advertiser and consumer behavior in response to the increased saliency of privacy considerations on the Internet. As a result, our estimates can be viewed as measuring the overall impact of the policy.

\section{Consumer Response to GDPR}

In this section we quantify the extent to which consumers utilize the GDPR-mandated ability to opt out. We measure how GDPR opt-out impacts the total number of cookies and searches observed by the intermediary. We then explore whether there were any changes in the composition of the remaining consumers who opted in.

\subsection{Opt-Out Usage}

Recall that we do not directly observe opt-out in our dataset because consumers who opt out are no longer part of our dataset. As a result, at time $t$, the total number of consumers on a website $j$ is given by the true number of consumers subtracted by the number of consumers who have opted out. ${ }^{30}$

$$
U_{j t}^{O B S}=U_{j t}^{T R U E}-U_{j t}^{O P T-O U T}
$$

In the control group, $U_{j t}^{O P T-O U T}=0$, whereas post-GDPR $U_{j t}^{O P T-O U T} \geq 0$. We assume parallel trends in $U_{j t}^{T R U E}$, which means that any change in $U_{j t}^{O B S}$ allows us to identify $U_{j t}^{O P T-O U T} .^{31,32}$

considerable number for which we are uncertain when they implemented the policy. Note that we do not drop the non-compliant websites from the treatment group since we are broadly interested in the overall impact of the policy.

${ }^{30}$ Note that a website here serves as a first-party affiliate of our intermediary; so the true number of consumers for website $j$ is not the true number of consumers for the intermediary, as opt-out consumers become out of its reach.

${ }^{31}$ As noted in Goldberg, Johnson and Shriver (2021), another possible complication is that this could be a result of firms changing the type of data that they send to third party services. To our knowledge there is no change in the data the websites send to the intermediary as a result of GDPR since the intermediary and the data are crucial for generating advertising revenue for these websites. Furthermore, if a website decided to stop using the intermediary altogether then, as noted previously, they would not be part of our sample.

${ }^{32}$ Another possible confounding factor is the sales activity of the intermediary. For instance, it's possible for the intermediary to sell additional advertising units to a website that can appear on pages of the website where the intermediary previously was not tracking before. If there was a differential effect from this around the date of the treatment then this could systematically bias the number of unique cookies and searches that we observe. To test the plausibility of this hypothesis, we run our difference-in-differences specification with total advertising units and total pages on which the intermediaries advertising appears as the outcome variables. The results in Table 6 show that there was no significant change in either of those two variables. Thus, we rule this alternative explanation out. 
Figure 4 displays the total unique cookies for two multi-national websites, one of which implemented the consent guidelines of the GDPR and the other which does business in the EU but did not immediately comply with the regulations. The multi-national website which implemented the consent guidelines shows a clear drop in observed cookies on European websites at the onset of GDPR. Columns (1) and (2) of Table 1 report the result of regression (1) with total number of observed unique cookies as the outcome variable. We consider the specification in both levels and logs. The estimates show that, in aggregate, GDPR reduced the total number of unique cookies by around $12.5 \%$. As previously mentioned, our estimates should be interpreted in the context of mixed compliance with the consent guidelines of GDPR as evidenced by Figure 4 .

It is important to note that this result does not imply that $12.5 \%$ of consumers made use of the opt-out features. This is because the unit of observation is a cookie, rather than a consumer. A single consumer can appear under multiple cookie identifiers if they make use of the aforementioned browser-based privacy means.

Another measure of consumer response is the total number of searches that are recorded by the intermediary. This outcome measure can also be interpreted as the overall data size observed by the intermediary and how it is affected by GDPR. We re-run the same specification with recorded searches as the dependent variable and report the results in columns (3) and (4) of Table 1 . We find that there's a $10.7 \%$ drop in the overall recorded searches which is qualitatively consistent with the effect size of the specification using the number of unique cookies.

In order to provide evidence for the validity of the difference-in-differences strategy we rely on our time-varying treatment specification. Figure 8 displays the resulting treatment effect over time and points to parallel pre-trends as well as a consistent treatment effect size over our sample period though there is a slight decrease in the estimated treatment effect as we approach the end of our sample period. Finally, as further evidence of robustness, we employ a synthetic control approach, which is reported in subsection B.1 and produces qualitatively similar results.

We want to discuss two further potential threats to the validity of our empirical strategy. The first is a potential contamination between treatment and control groups that may result from multi-national companies implementing the consent mechanisms across all of their websites. The second is that the results may be driven by seasonal travel differences between the treatment and control groups. The first is not a big concern in our setting because multi-national online travel agencies serve customers through country-specific websites and have incentives to only make their EU domains compliant with GDPR. For the online travel agencies where we can directly verify compliance we do indeed see that most of them only implement it for their respective EU domains as evidenced by Figure 4. Furthermore, to the extent that there is still residual contamination, it would mean that our estimates are a lower bound of the true effect size.

For the second issue, we focus our analysis on a tight window around the GDPR implementa- 
tion date and select control countries that ought to have similar travel patterns during this time period. However, since European travel patterns have a somewhat steeper summer gradient than US travel patterns we would expect this to bias against our results. We therefore further supplement our analysis with Google Trends data on travel searches, which should be unaffected by GDPR and provide a good picture into travel trends across these different countries. Using this data, we first graphically show that in the period of the year that we consider, travel patterns between the countries in the analysis are similar. When we augment our primary analysis with country-specific seasonal controls based on Google Trends data we find quantitatively very similar results with slightly stronger effect sizes than before. The full details of this exercise are deferred to subsection B.2.

\subsection{Persistence of Identifier}

A natural question is whether GDPR affects the ability to persistently track consumers. To address this question, we define an identifier persistence measure that tracks how often cookies that we see in a given week return after $k$ weeks, where we explore different values for $k(1,2,3$, and 4 weeks). Let $C_{j t}$ be the set of cookies seen in week $t$ on website $j$, the measure is then given by:

$$
\text { persistence }_{k, t}=\frac{\left|C_{j, t} \cap C_{j, t+k}\right|}{\left|C_{j, t}\right|}
$$

In Figure 5 we set $k=4$ and display the persistence measure for the same two multi-national websites with country-specific versions of their website over time. At the onset of GDPR there is a clear increase in persistence on the EU-based websites, but no noticeable difference in the nonEU websites. We further validate this increase by running our baseline difference-in-differences specification using the persistence outcome variable for $k \in\{1,2,3,4\} .33,34$

Table 2 shows the results of this regression, which indicate that there is a statistically significant and meaningful increase in consumer persistence and that this effect gets more pronounced as $k$ increases. ${ }^{35}$ We further run the time-varying treatment specification (2) in order to validate that parallel trends holds and to understand the consistency of the effect over time.

\footnotetext{
${ }^{33}$ In order to run specification (1) we drop the last 4 weeks of our sample so that we are utilizing the same sample as we vary $k$. However, our results are qualitatively robust to including these weeks when the data for them is available.

${ }^{34}$ Note that the units on the regression and Figure 5 are not the same. Figure 5 displays the persistence measure in terms of percent deviations from week 20 whereas the coefficients in Table 2 are changes in levels.

${ }^{35}$ It is important to note that the persistence measure may have some noise when $k=1$ due to consumer activity near the end of the week that spills over into the next week and falsely appears as persistence. As a result, the most reliable measures of consumer persistence are for $k \geq 2$, but we report $k=1$ for completeness.
} 
Figure 4: Total Number of Unique Cookies for Two Multi-National Website.
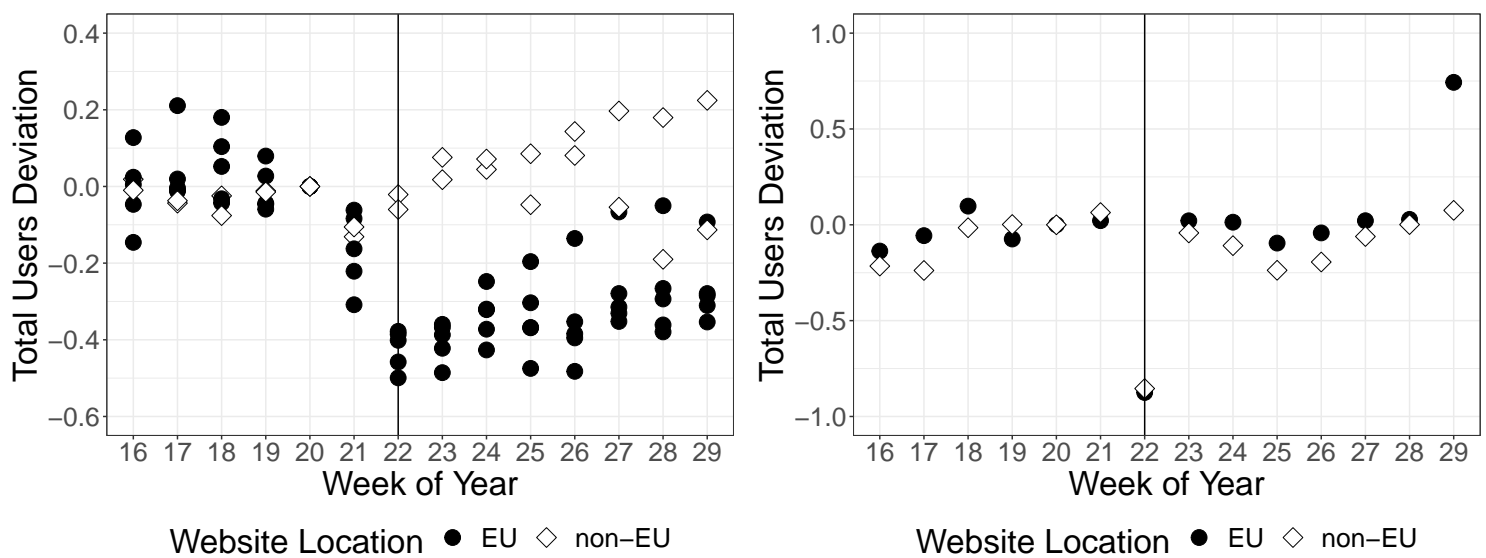

Notes: Each point on the graph represents the total number of unique cookies for a single country, reported in terms of its percent deviation relative to week 20, or $\frac{U_{t}-U_{t=20}}{U_{t=20}} \forall t \neq 20$. The figure on the left presents a multi-national website that we were able to verify implemented the consent guidelines of GDPR. In this figure, the black dots represent the represented European countries (United Kingdom, France, Germany, Italy, Spain) and the two white dots represent the two non-EU countries where this website functions - the United States and Canada. The figure on the right presents a multi-national website that we were able to verify did not implement the consent guidelines of GDPR. The black dots represent the values from the United Kingdom and the white dots represent the values from the United States.

Table 1: Difference-in-Differences Estimates for Cookies and Searches

\begin{tabular}{lcccc}
\hline & $(1)$ & $(2)$ & $(3)$ & $(4)$ \\
& $\begin{array}{c}\log (\text { Unique } \\
\text { Cookies) }\end{array}$ & $\begin{array}{c}\text { Unique } \\
\text { Cookies }\end{array}$ & $\begin{array}{c}\log \text { (Recorded } \\
\text { Searches) }\end{array}$ & $\begin{array}{c}\text { Recorded } \\
\text { Searches }\end{array}$ \\
\hline DiD Coefficient & $-0.125^{* *}$ & $-1378.1^{*}$ & $-0.107^{*}$ & $-9618.3^{* *}$ \\
& $(-2.43)$ & $(-1.71)$ & $(-1.87)$ & $(-2.24)$ \\
Product Type Controls & $\checkmark$ & $\checkmark$ & $\checkmark$ & $\checkmark$ \\
OS + Browser Controls & $\checkmark$ & $\checkmark$ & $\checkmark$ & $\checkmark$ \\
Week FE & $\checkmark$ & $\checkmark$ & $\checkmark$ & $\checkmark$ \\
Website $\times$ Country FE & $\checkmark$ & $\checkmark$ & $\checkmark$ & $\checkmark$ \\
\hline Observations & 63840 & 63840 & 63840 & 63840 \\
\hline
\end{tabular}

${ }^{*} p<0.10,{ }^{* *} p<0.05,{ }^{* * *} p<0.01$

Notes: t-statistics are reported in parentheses. The standard errors for every regression are clustered at the website-country level. We aggregate every dependent variable to the website-country-browser-OS-product type-week level between weeks 16 and 29, including both weeks 16 and 29 (April 13th - July 20th). The dependent variables in the regression reported in the first and second column are the log and overall level of the number of unique cookies observed. The dependent variables in the regression reported in the third and fourth column are the log and overall level of the number of total recorded searches. 
Figure 10 shows that while for $k=1$ the time dependent treatment effects are more noisy, for all $k \geq 2$ parallel trends hold and the treatment effect is stable over time. ${ }^{36}$ The treatment effect remains roughly the same as $k$ grows, even though Table 5 shows that the mean persistence declines as $k$ increases. For instance, in the pre-treatment period, the mean persistence for EU websites was 0.0597 and the estimated treatment effect is 0.005 indicating a roughly $8 \%$ increase in persistence.

Recall from subsection 2.2 that there are two plausible mechanisms for driving this increase in persistence: privacy means substitution and selective consent. While it is hard to fully disentangle the two explanations, we are able to provide suggestive evidence that both play a role. For that we focus on a large website that has faithfully implemented a consent policy. While both mechanisms imply that the drop in relative probability mass should be concentrated at the lower end of the support, in our context one signature of browser-based privacy protection is a large mass of "single search" consumers. This is because advertising/cookie blockers interact with the identifier set by the intermediary by continually regenerating it after every search as discussed in section 2. Indeed, Figure 6 shows that the fraction of single searchers significantly dropped after the implementation of GDPR and the relative probability mass of searchers with more than one search increased. While a disproportionate drop in single searchers is suggestive of a larger role for privacy means substitution, it is still plausible that part of this could be driven by selective consent.

To test how privacy means substitution and selective consent contribute to the observed changes, we set up a simple model (for details see Appendix D). The model generates the distribution of search histories as a mixture of obfuscators, who always generate search histories of length one, and non-obfuscators, whose history is captured by a parametric count distribution. Under this formalization, the "excess" number of single searchers identifies the fraction of obfuscators and the residual count distribution the true underlying search histories. We can use a Vuong test to determine whether obfuscators are needed to fit the observed distribution (Vuong, 1989). We use the test to determine the presence of obfuscators and whether the fraction changes with the introduction of GDPR. In addition, we can test for whether true search histories are significantly longer after GDPR comes into effect, which is indicative of selective consent. ${ }^{37}$

\footnotetext{
${ }^{36}$ Furthermore, Figure 9 in the appendix shows the overall distributions of consumer persistence for the EU vs. non-EU and note that there are some outliers. In particular, there is a large mass of high persistence observations and persistence measures close to 0 . Our results are qualitatively robust to running our specifications winsorizing and dropping these observations as well. They are also robust to the addition of seasonal travel controls using the same procedure as in subsection B.2.

${ }^{37}$ One caveat with this model is that consumers that delete their cookies after several searches are not considered obfuscators.
} 
Figure 5: Four Week Persistence for Two Multi-National Websites
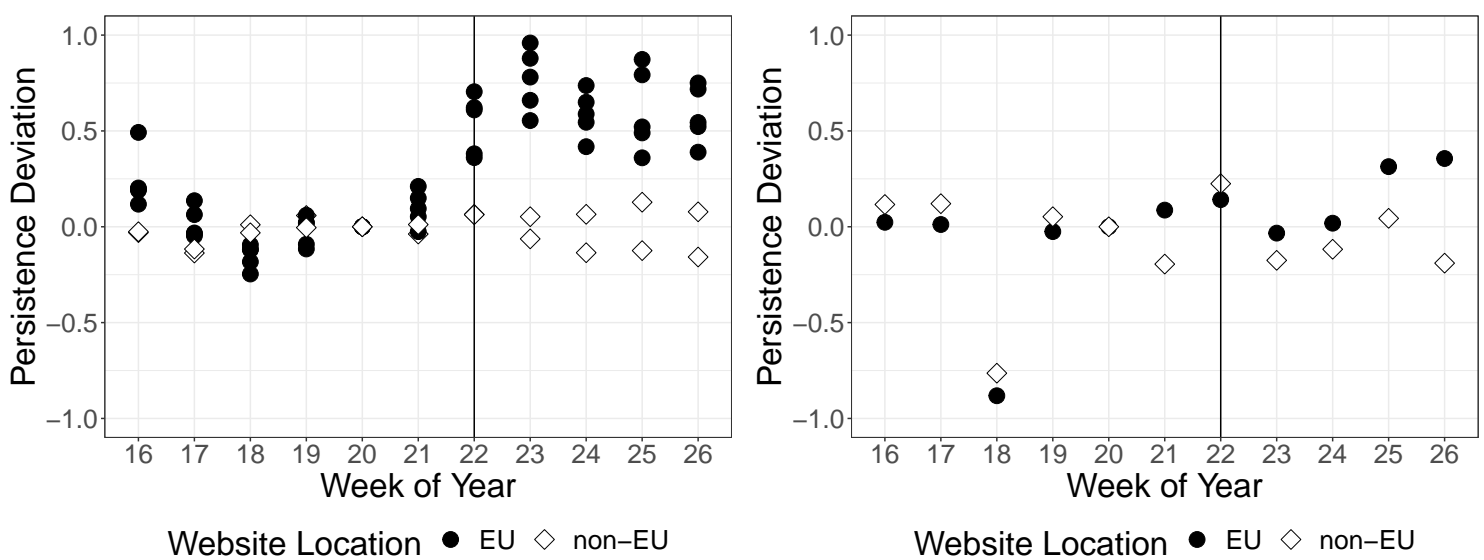

Notes: Each point on the graph represents the four week persistence fraction for a single country, reported in terms of

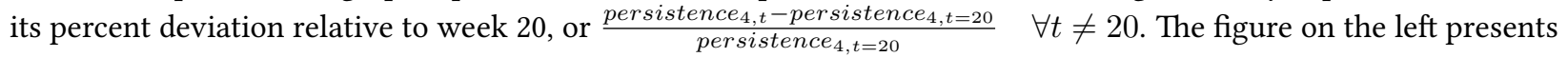
a multi-national website that we were able to verify implemented the consent guidelines of GDPR. In this figure, the black dots represent the represented European countries (United Kingdom, France, Germany, Italy, Spain) and the two white dots represent the two non-EU countries where this website functions - the United States and Canada. The figure on the right presents a multi-national website that we were able to verify did not implement the consent guidelines of GDPR. The black dots represent the values from the United Kingdom and the white dots represent the values from the United States.

Table 2: Difference-in-Differences Estimates for Consumer Persistence

\begin{tabular}{lcccc}
\hline & $(1)$ & $(2)$ & $(3)$ & $(4)$ \\
& 1 Week & 2 Weeks & 3 Weeks & 4 Weeks \\
& Persistence & Persistence & Persistence & Persistence \\
\hline DiD Coefficient & $0.00308^{*}$ & $0.00416^{* * *}$ & $0.00382^{* * *}$ & $0.00505^{* * *}$ \\
& $(1.96)$ & $(3.40)$ & $(3.10)$ & $(3.50)$ \\
Product Type Controls & $\checkmark$ & $\checkmark$ & $\checkmark$ & $\checkmark$ \\
OS + Browser Controls & $\checkmark$ & $\checkmark$ & $\checkmark$ & $\checkmark$ \\
Week FE & $\checkmark$ & $\checkmark$ & $\checkmark$ & $\checkmark$ \\
Website $\times$ Country FE & $\checkmark$ & $\checkmark$ & $\checkmark$ & $\checkmark$ \\
\hline Observations & 50160 & 50160 & 50160 & 50160 \\
\hline
\end{tabular}

${ }^{*} p<0.10,{ }^{* *} p<0.05,{ }^{* * *} p<0.01$

Notes: $\mathrm{t}$-statistics are reported in parentheses. The standard errors for every regression are clustered at the website-country level. We aggregate every dependent variable to the websitecountry-browser-OS-product type-week level between weeks 16 and 26, including both weeks 16 and 26 (April 13th - June 29th). The dependent variables in the regression are the consumer persistence measures for $k=1,2,3,4$, respectively. 
Figure 6: Change in Search Distribution for One Site
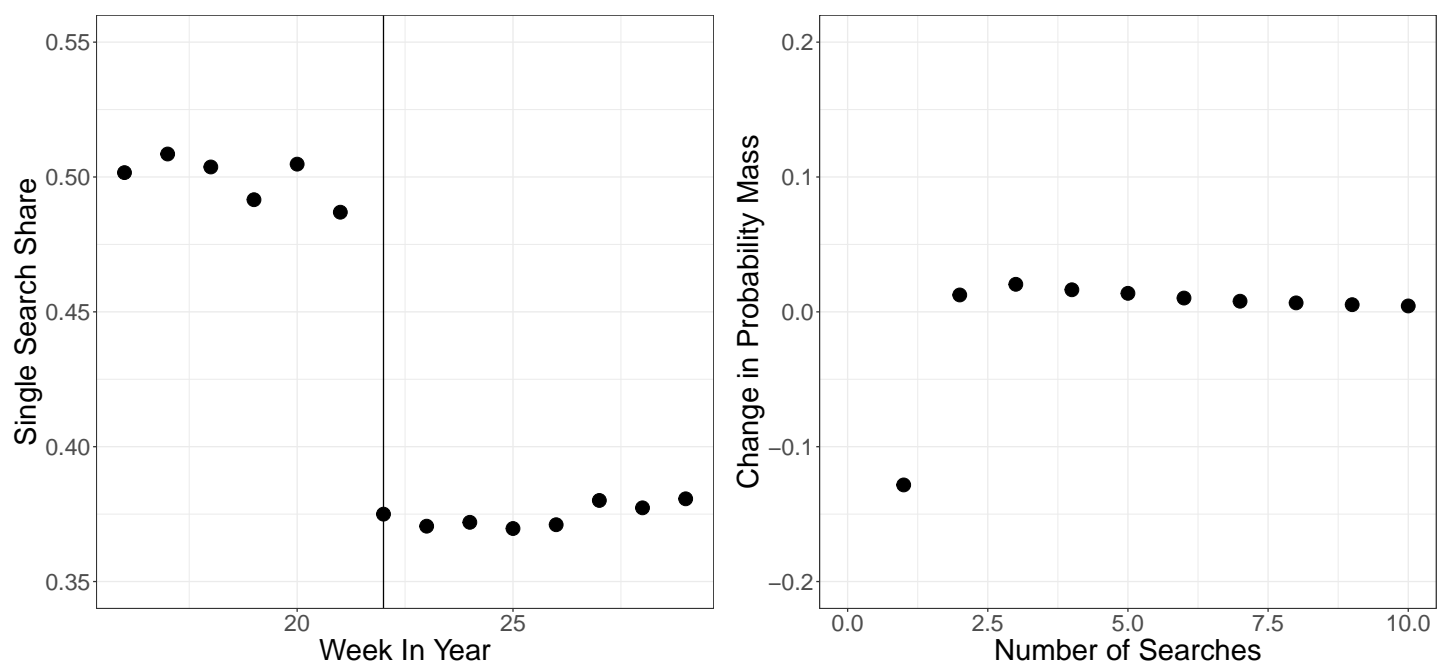

Notes: The figure on the left breaks down the share of cookies associated with only one search week by week, as opposed to pooling the full sample periods before and after GDPR. The figure on the right shows the difference in the share of consumers with $x$ searches in the full sample after GDPR compared to before GDPR. For instance, the leftmost point indicates that there was a roughly $12.8 \%$ decrease in the share of cookies associated with a single search.

We estimate the model under two different distributional assumptions for the count distribution of the true number of searches. First, we assume a conditional Poisson distribution and, second, we assume that it is negative binomial, to allow for more dispersion. In short, we find evidence for both of the mechanisms as the test suggests the presence of obfuscators in the preGDPR period but not in the post-GDPR period, while also showing that the average length of the (true) underlying search history increased slightly.

We can additionally test for the presence of selective consent by investigating whether large and small websites exhibit heterogeneous treatment effects. ${ }^{38}$ Selective consent implies that persistence should increase more for large websites. The results of this regression are reported in Table 7, which points to a larger, but not statistically significant, increase in persistence for larger websites. We interpret this as weak evidence for the presence of selective consent.

Finally, we investigate heterogeneous treatment effects across popular web browsers and operating systems. We find that the increase in persistence occurs on all browsers except for Internet Explorer and suggestive evidence that it is more pronounced on desktop compared to mobile operating systems. These differences are plausible under privacy means substitution, in light of the alleged lack of technical sophistication of Internet Explorer users and the technical difficulty

\footnotetext{
${ }^{38}$ We define a "large" website as a website above the median size and a "small" website as a website below the median size. Recall from section 2 that the consent decision is elicited when the consumer visits the website with essentially no information about the intermediary that we partner with. As a result, the most relevant degree of heterogeneity for selective consent decision is the size of the first party website.
} 
of utilizing browser-based privacy means on mobile devices and the Internet Explorer. ${ }^{39}$ However, the results between other browsers and operating systems are less clear cut which suggests that selective consent is also driving the results. The results and a full discussion are deferred to Appendix C.

Overall, these results provide robust evidence for privacy means substitution driving the increase in persistence, while also some degree of selective consent.

\section{GDPR and Online Advertising}

The advertisements in our setting are sold by the intermediary on the host OTA website via real-time auctions that are held when a consumer makes a search query. ${ }^{40}$ Advertisers, who are typically competing OTAs or airlines, bid on search keywords such as the origination, destination, or dates of travel. For example, an advertiser may submit a bid to show an advertisement for a consumer searching for a flight from JFK to LAX and upon winning displays a price comparison advertisement for this same travel itinerary. Thus, bids are shaped by the average value of attracting a consumer to the advertisers' website for the same itinerary. ${ }^{41}$ Bids are submitted per click and a payment from the advertiser to the intermediary occurs only if the consumer clicks on the advertisement. An important fact for the interpretation of our results is that consumers who opt out are never shown any advertisements. Thus, the intermediary generates no advertising revenues from these consumers. ${ }^{42}$

We separately investigate the changes in advertising revenue, prices, and quantity of advertisement. First, we look for the change in the number of clicks for advertisements following GDPR. Columns (1) and (2) of Table 3 show that there is a statistically significant decrease of $13.5 \%$ in the total number of clicks. The magnitude of this effect is in line with the drop in total

\footnotetext{
${ }^{39}$ In the time period of interest, the new Microsoft Edge browser was the default on Windows computers and Internet Explorer is predominantly utilized on computers running the Windows OS. Microsoft Edge was the default on Windows since 2015, thus users of Internet Explorer are predominantly those on older computers. Internet Explorer users tend to be older than Chrome or Firefox users (Internet Explorer Users are Older - Retrieved on May 27th, 2022) and thus less inclined to adopt browser-based privacy practices (Zou et al., 2020).

${ }^{40}$ The auction format is a linear combination of a generalized first and second price auction where there are $N$ advertisers and $k$ slots.

${ }^{41}$ The fact that the advertisers are bidding to direct the consumer to their website for the same search differs from the standard sponsored search auction in which the same product may be sold under many keyword searches and categories, which makes conversion and attribution difficult. In particular, it means that unlike standard keyword search advertising, it is clear whether an ad converted into purchase so long as a third-party cookie allows the advertiser to link the attributed purchase to the advertisement placed on the host website. Thus, the only possible changes to the ability to do attribution in this setting would be through changes in the ability to track the average consumer. Importantly, this means that advertisers do not bid on particular consumer histories, so any observed changes come from a change in the overall composition of consumers.

${ }^{42} \mathrm{An}$ implication of this is that although GDPR opt-out restricts the data observed by the intermediary and the website, we observe the advertising revenue for the intermediary generated from opt-in consumers.
} 
cookies and searches. We next look for changes in the number of clicks from distinct cookies to see if any changes were driven by some small set of consumers. Columns (3) and (4) show that this measure also decreases significantly. Figure 7 displays the time-varying specification for these outcome variable and shows that the effect on the number of clicks is relatively constant.

Columns (5) and (6) of Table 3 show the effects on revenue. The magnitude of the point estimates suggests an economically significant drop, though it is imprecise and not statistically significant. The time-varying treatment effect displayed in Figure 7 shows that revenue initially falls sharply after the implementation of GDPR and then begins to slightly recover. Importantly, column (7) of Table 3 shows that the average bid of the advertisers increases. At roughly $12 \%$ this increase is economically sizable. ${ }^{43}$ The time-varying coefficient in Figure 7 shows that the average bid does not change initially after the policy and then increases gradually. In summary, the immediate drop in clicks following GDPR leads to a sharp drop in revenue, but the gradual increase in the average bids leads to a recovery of some of the lost revenue for the intermediary and advertisers.

In light of these results one may wonder how the quantity of advertisements is affected. Using the same difference-in-differences specification with total number of advertisements as the dependent variable we find that the number of advertisements has dropped but that this change is not significant (see Table 11 and Figure 13 in Appendix E for the time varying treatment effect).

We now discuss the plausible mechanisms behind the increase in prices (winning bids). The first mechanism, which is consistent with the evidence that we establish above, is that remaining consumers are of higher average value to advertisers. ${ }^{44}$ Our discussion with the intermediary indicates that advertisers' value is determined according to the observed conversion rate of their advertisements, which is the fraction of consumers that end up purchasing a good after clicking on an advertisement. Consistent with our explanation in subsection 2.2, it is plausible that as a result of privacy means substitution the increased trackability of consumers following GDPR improved the measurement of conversion rates, thus contributing to an increase in value of consumers as perceived by the advertisers. It is further important to recall from subsection 2.2 that the observed increase in prices is inconsistent with the predictions of selective consent.

There are several pieces of evidence supporting privacy means substitution. First, before GDPR $24.9 \%$ of advertisements on flight searches are targeting consumers whose cookie history is of length one, which indicates that this group is quantitatively important for advertisers.

\footnotetext{
${ }^{43}$ Note that in order to preserve the privacy of our intermediary, the bid and revenue values are obfuscated by a common multiplier. However, the interpretation of percentage changes is preserved under this transformation. The average obfuscated bid value in the EU pre-GDPR was 126.9.

${ }^{44}$ In contemporary work and in a different e-commerce setting, Goldberg, Johnson and Shriver (2021) reach a similar conclusion about the value of consumers post-GDPR.
} 
Figure 7: Week by Week Treatment Effect for Total Clicks, Revenue, and Average Bid asinh(Total Clicks)
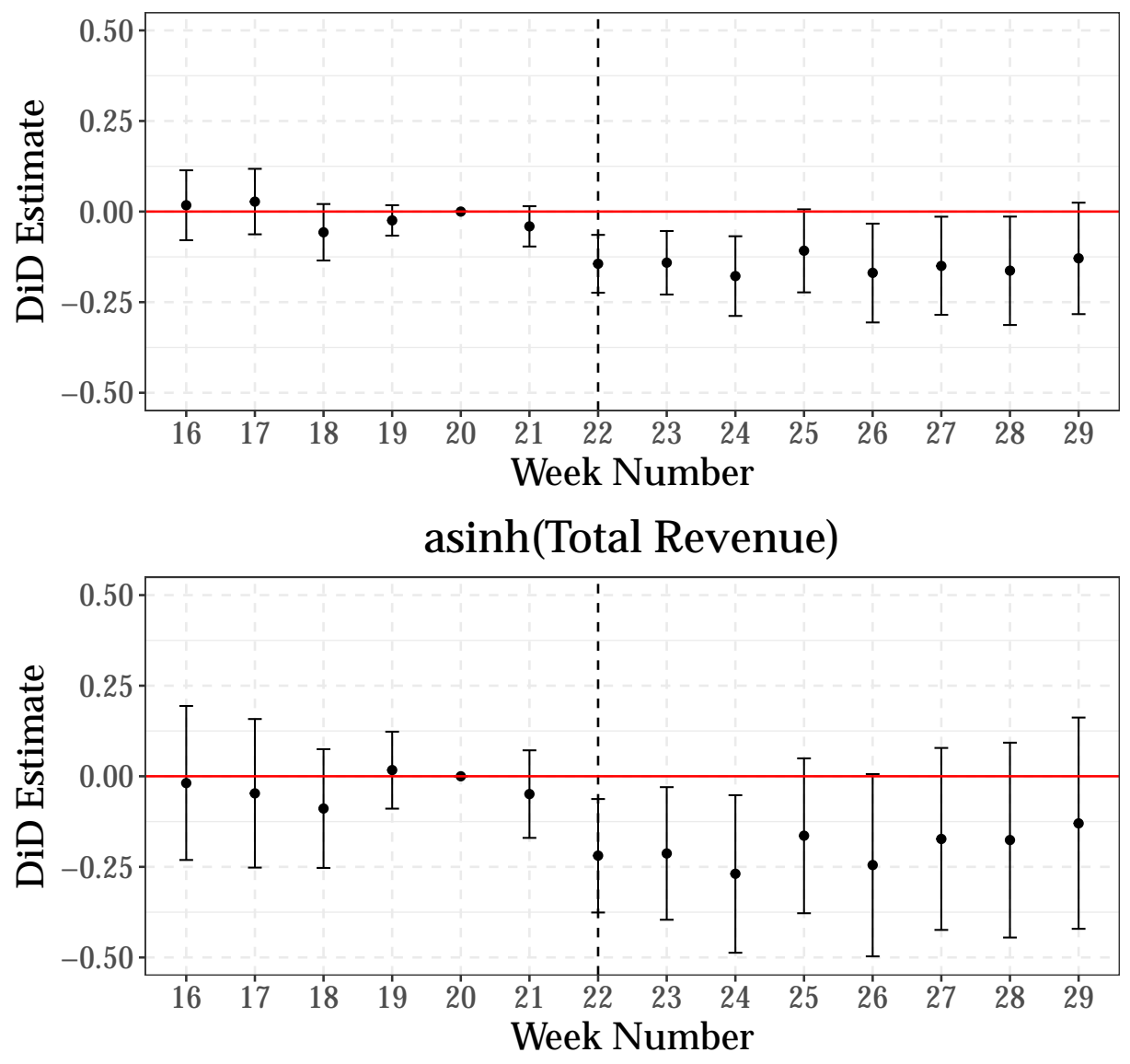

Average Bid

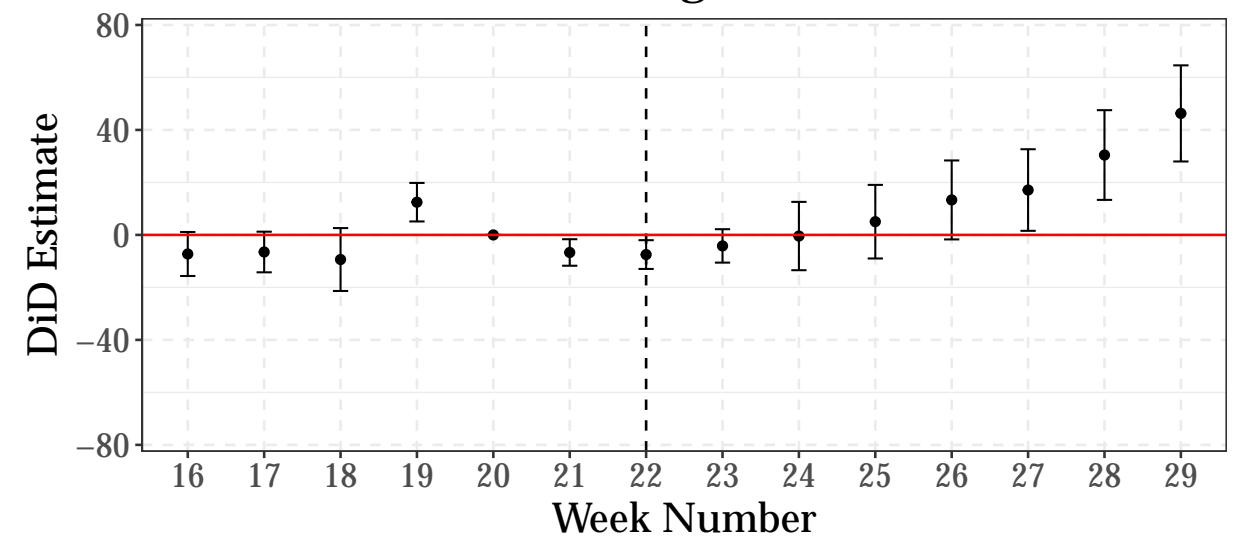


Table 3: Difference-in-Differences Estimates for Advertising Outcome Variables

\begin{tabular}{|c|c|c|c|c|c|c|c|}
\hline & (1) & (2) & (3) & (4) & (5) & (6) & (7) \\
\hline & asinh(Total & Total & $\operatorname{asinh}$ (Distinct & Distinct & & & Average \\
\hline & Clicks) & Clicks & Clicks) & Clicks & asinh(Revenue) & Revenue & Bid \\
\hline \multirow[t]{2}{*}{ DiD Coefficient } & $-0.135^{* *}$ & $-251.9^{*}$ & $-0.133^{* *}$ & $-214.9^{*}$ & -0.168 & -32972.3 & $15.41^{* * *}$ \\
\hline & $(-2.32)$ & $(-1.91)$ & $(-2.33)$ & $(-1.84)$ & $(-1.54)$ & $(-0.75)$ & $(2.90)$ \\
\hline OS + Browser Controls & $\checkmark$ & $\checkmark$ & $\checkmark$ & $\checkmark$ & $\checkmark$ & $\checkmark$ & $\checkmark$ \\
\hline Product Category Controls & $\checkmark$ & $\checkmark$ & $\checkmark$ & $\checkmark$ & $\checkmark$ & $\checkmark$ & $\checkmark$ \\
\hline Website $\times$ Country FE & $\checkmark$ & $\checkmark$ & $\checkmark$ & $\checkmark$ & $\checkmark$ & $\checkmark$ & $\checkmark$ \\
\hline Week FE & $\checkmark$ & $\checkmark$ & $\checkmark$ & $\checkmark$ & $\checkmark$ & $\checkmark$ & $\checkmark$ \\
\hline Observations & 62328 & 62328 & 62328 & 62328 & 62328 & 62328 & 62328 \\
\hline
\end{tabular}

${ }^{*} p<0.10,{ }^{* *} p<0.05,{ }^{* * *} p<0.01$

Notes: $\mathrm{t}$-statistics are reported in parentheses. The standard errors for every regression are clustered at the website-country level. We aggregate every dependent variable to the website-country-browser-OS-product type-week level between weeks 16 and 29, including both weeks 16 and 29 (April 13th - July 20th). The dependent variable in the regression reported in the first column is the total number of clicks associated with each observation and the second column is the inverse hyperbolic sine transform of this value. Likewise, the dependent variables in the third and fourth columns are the total number and inverse hyperbolic sine transform of the total number of unique cookies who interacted with advertisements. The dependent variables in the fifth and sixth column are the total number and inverse hyperbolic sine transform of the total revenue. The dependent variable in the seventh column is the average bid by advertisers. We utilize the inverse hyperbolic sine transform instead of the logarithm as in previous sections as some of the outcome variables we consider in this section can take zero values. The inverse hyperbolic sine transform is given by $\bar{y}=\operatorname{arcsinh}(y)=\ln \left(y+\sqrt{y^{2}+1}\right)$ and results in a similar coefficient interpretation as taking logarithms Bellemare and Wichman (2019), but does not remove the zero valued observations from the data. We retain the zero values here so that there is a clearer comparison between the estimates before and after the transformation. 
Second, we find that the fraction of consumers searching for flights who receive advertisements has increased, which is consistent with the explanation that the average remaining consumer has become more valuable (see Table 12). One possible objection to this interpretation may be that the two types of consumers systematically differ in their search and purchase patterns, resulting in differences in value to advertisers based on the types of flights that they buy. However, we have no indication that users with single cookie histories search for flights of different value. For instance, we find that their average days to departure when searching and fraction of searches that are for domestic flights are extremely similar to consumers with longer histories (see Table 13 and Table 14). Thus, it is likely that the set of remaining consumers are equally valuable and the price increase is due to a higher chance of attribution.

However, there might be two plausible alternative explanations. The first is that GDPR has decreased the "supply" of consumers to whom advertisements can be served. As we have demonstrated above, there is a significant reduction in the number of advertisements served because consumers opt out. This reduction in advertising targets might increase the value of advertisement to the remaining consumers. While this is certainly plausible, the pattern of price increases is not fully consistent with the supply shock explanation. Since this shock materializes right after the implementation date and advertising budgets are set daily, one would expect a sharp price increase. Instead, we observe a gradual price increase (Figure 7), which is more consistent with advertisers slowly adjusting to the equally slow increase in conversion rates.

Another plausible alternative explanation is that GDPR is a positive "demand shock" for the type of advertising offered by the intermediary. Advertisers in our setting submit bids based on the context in which advertising is shown (e.g. based on travel search details) instead of on individual consumer histories. The relative efficiency of such "contextual advertising" compared to behaviorally targeted display advertising, which is even more dependent on consumer tracking, may have increased as a result of GDPR. ${ }^{45}$ However, our setting also contains a personalized element: the decision to place advertisements is personalized based on the predictions of the intermediary. It is therefore less plausible that the intermediary would be one of the clear-cut winners under such a shift in the market. We also provide two pieces of direct evidence that the change in prices is not predominantly driven by changes in demand. First, we find that the number of bidders in the advertising auctions of the intermediary has not increased and in fact slightly decreased (Column (5) in Table 16). While this rules out a positive demand shock at the extensive margin, it could still be that specific advertisers purchase more advertisement than usual, perhaps because of a big campaign. In order to explore whether this was the case we compute the share of winning bids for each advertiser and construct common measures of buyer concentration - HHI

\footnotetext{
${ }^{45}$ Digiday - Personalization diminished: In the GDPR era, contextual targeting is making a comeback. Retrieved on December 15th, 2020.
} 
and concentration ratios. While we find a statistically significant increase in concentration, this effect is economically small (Columns (1)-(4) in Table 16). For instance, the increase in market concentration of the largest five advertisers per website $(C R-5)$ is roughly $3 \%$ (see Table 15 ).

To sum up, it seems plausible that advertising prices, at least in part, increased because the average consumer is more trackable. This interpretation is in line with the evidence of previous sections and alternative explanations are less plausible based on our institutional knowledge and prevailing patterns in the data. However, we must caution that the evidence that we provide is not conclusive about the mechanism and that it is known that inference in such niche advertising markets may be driven by large advertisers. Nonetheless, our results in this section suggest the importance of understanding the interplay between advertising attribution and privacy regulation.

\section{GDPR and Prediction of Consumer Behavior}

In this section we investigate whether the changes due to GDPR have affected the intermediary's ability to predict consumer behavior. Beyond this particular context, such an investigation is also of broader interest. Sophisticated machine learning technologies that attempt to predict consumer purchase behavior are becoming increasingly common and our results provide a case study on how their accuracy is affected by data privacy regulation. ${ }^{46}$

Based on our analysis we expect there to be three predominant reasons why we might observe a change in the ability to predict. First, GDPR has significantly reduced the overall amount of data. Second, remaining consumers have longer histories and are more trackable. Third, in line with our illustrations in Figure 2 and Figure 3, GDPR might reveal correlation structures between consumer behavior and the length of consumer histories that were previously obfuscated by the use of alternative privacy means. We would expect the first effect to decrease prediction performance and the second and third to increase prediction performance.

We take as given both the setup of the prediction problem and the algorithm that the intermediary uses. This allows us to understand the effects of GDPR on the prediction problem "in the field." Its problem is to predict whether a consumer will purchase from the current site based on the history that the intermediary observes about this consumer on the current website. Specifically, its algorithm classifies a search by a consumer into two categories: purchasers and non-purchasers, based on whether the consumer will purchase a product on the current website within some time

\footnotetext{
${ }^{46}$ See, for example, Wall Street Journal - Retailers Use AI to Improve Online Recommendations for Shoppers. Retrieved on March 31st, 2021.
} 
window. Formally, each query is classified into

$$
y_{i, j, k}= \begin{cases}1, & \text { if } i \text { is a purchaser on website } j \text { after search } k \\ 0, & \text { if } i \text { is not a purchaser on website } j \text { after search } k\end{cases}
$$

for a consumer $i$ on website $j$ on the $k$ th query observed by the intermediary. We denote the classification made in real-time by the intermediary as $\hat{y}_{i, j, k}$. For every consumer $i$ we observe a series of searches on website $j, X_{i, j, 1}, X_{i, j, 2}, \ldots, X_{i, j, n}$ and, if the consumer ended up making a purchase on this website, the timestamp of when consumer $i$ purchased on website $j$. This allows us to further construct the ground truth label, $y_{i, j, k}^{T R U E}$, which we use to evaluate the performance of the classifier. ${ }^{47}$ We will denote the class proportion as the proportion of searches whose ground truth label is purchaser.

For each search, the intermediary produces a probability estimate that the consumer is a purchaser:

$$
p_{i, j, k}=\operatorname{Pr}\left(y_{i, j, k}^{T R U E}=1 \mid X_{i, j, 1}, \ldots, X_{i, j, k}\right), \forall i, j, k
$$

We observe the intermediary's predicted $\hat{p}_{i, j, k}$ and $\hat{y}_{i, j, k}$ for every search as well as the $y_{i, j, k}^{T R U E}$ which we construct. The conversion of probability estimate, $\hat{p}_{i, j, k}$, to actual classification, $\hat{y}_{i, j, k}$, is based on whether the consumer's "score", $\hat{p}_{i, j, k}$, is above or below a chosen threshold $\hat{P}$. The threshold is chosen based on revenue considerations and other factors irrelevant to the quality of the predictions and, as a result, we focus on analyzing the prediction error associated with the probabilistic estimate $\hat{p}_{i, j, k}$ and not $\hat{y}_{i, j, k}$.

\subsection{Prediction Evaluation Measures}

To evaluate the performance of the classifier deployed by the intermediary, we use two standard measures from the machine learning literature: the Mean Squared Error (MSE) and Area under the ROC Curve (AUC).

The MSE computes the mean of the squared errors associated with the predicted estimate $\hat{p}_{i, j, k}$ relative to the realized binary event. Specifically, let $\mathcal{I}_{j}$ be the set of all consumers on website $j$ and let $\mathcal{K}_{i, j}$ be the set of all events for consumer $i$ on website $j$. Then, the MSE of website $j$ is

\footnotetext{
${ }^{47}$ The ground truth labels are constructed by setting $y_{i, j, k}^{T R U E}=1$ if the purchase occurs within $N_{j}$ days of the search and $y_{i, j, k}^{T R U E}=0$ otherwise. While in practice the value of $N_{j}$ is website-dependent, we do not observe this value for each website so we restrict focus to $N_{j}=2$ across all websites. For the majority of websites in our sample, the intermediary informed us that they set $N=1$ or $N=2$. Furthermore, from our preliminary analysis, the results do not qualitatively differ between $N=1$ and $N=2$.
} 
given by,

$$
M S E_{j}=\frac{1}{\sum_{i \in \mathcal{I}_{j}}\left|\mathcal{K}_{i, j}\right|} \sum_{i \in \mathcal{I}_{j}} \sum_{k \in \mathcal{K}_{i, j}}\left(\hat{p}_{i, j, k}-y_{i, j, k}^{T R U E}\right)^{2}
$$

with a low MSE indicating a good prediction performance.

Although commonly used, the MSE has a couple of drawbacks for the current purpose. First, the measure is sensitive to the skewness of, and the change in, the class distribution. In the current context, about $90 \%$ of the searches result in non-purchase, which means that the estimate $\hat{p}_{i, j, k}$ tends to be low; intuitively, the estimate would tolerate more errors associated with the "infrequent" event (purchase) in order to minimize the errors associated with the more "frequent" event (non-purchase). Suppose now the class distribution changes so that more searches result in purchases. This is indeed what happens in our data after GDPR. Then, even though the consumer may not have become less predictable, MSE would rise artificially, due to the convexity associated with the formula, especially if the prediction algorithm does not adjust to the change in the distribution. Second, perhaps not unrelated to the first issue, the MSE is not the measure that the intermediary focuses on for its operation as well as for communicating with its partners. Instead, it focuses on AUC (the area under the curve), which we now turn to.

The AUC measures the area under the Receiver Operating Characteristic (ROC) curve. ${ }^{48}$ The ROC curve in turn measures how well the classifier trades off Type I ("false positive") with Type II ("false negative") errors. The AUC provides a simple scalar measure of the prediction performance. If either the prediction technology improves or the consumer becomes more predictable, then the ROC will shift up and AUC will increase. Aside from the fact that the intermediary focuses on this measure, the AUC is invariant to the change in class distribution (Fawcett, 2006). Suppose for instance the proportion of purchasers increases. As long as the prediction technology remains unchanged the ROC and AUC remain unchanged.

These two measures capture different aspects: AUC captures the ability for the classifier to separate the two different classes whereas MSE captures the accuracy of the estimated probabilities. Hence, we will report the effect on both since they provide two qualitatively different measures of prediction performance.

\subsection{Prediction Performance}

In this section we investigate the impact of GDPR on predictability at the immediate onset of its implementation. We utilize the same empirical strategy that we described in section 3 . The same empirical design is valid because the intermediary trains separate models for each website using

\footnotetext{
${ }^{48}$ We provide additional details on the construction of the AUC and its interpretation in Appendix F.1.
} 
only the data from the respective website. As a result, any changes to the collected data from EU websites due to GDPR should not impact non-EU websites. However, there are two limiting factors in our analysis. The first is the restriction on the data; unlike the search and advertising data, the prediction performance requires additional purchase data, which is available only for a subset of websites. ${ }^{49}$ The second is that the models are trained utilizing a sliding window of the data, which means that, even if there is a sudden change to the underlying data distribution, there may be a slow adjustment period that would vary across the different websites. Since the pool of consumers has changed with GDPR, our predictability regressions compare the larger set of consumers before GDPR with a smaller set of consumers after GDPR. Changes in predictability are therefore a function of both the quantity of data and the selection of consumers where consumers with longer histories remain in the data.

Table 4: Difference-in-Differences Estimates for Prediction Outcome Variables

\begin{tabular}{lcccccc}
\hline & $(1)$ & $(2)$ & $(3)$ & $(4)$ & $(5)$ & $(6)$ \\
& Class & Average & & & Purchaser & Non-Purchaser \\
& Proportion & Predicted Probability & MSE & AUC & MSE & MSE \\
\hline DiD Coefficient & $0.00915^{*}$ & 0.00129 & $0.0130^{* * *}$ & 0.0124 & -0.00579 & -0.00126 \\
& $(1.77)$ & $(0.17)$ & $(3.74)$ & $(1.12)$ & $(-0.43)$ & $(-0.45)$ \\
Product Type Controls & $\checkmark$ & $\checkmark$ & $\checkmark$ & $\checkmark$ & $\checkmark$ & $\checkmark$ \\
OS + Browser Controls & $\checkmark$ & $\checkmark$ & $\checkmark$ & $\checkmark$ & $\checkmark$ & $\checkmark$ \\
Week FE & $\checkmark$ & $\checkmark$ & $\checkmark$ & $\checkmark$ & $\checkmark$ & $\checkmark$ \\
Website $\times$ Country FE & $\checkmark$ & $\checkmark$ & $\checkmark$ & $\checkmark$ & $\checkmark$ & $\checkmark$ \\
\hline Observations & 15470 & 15470 & 15470 & 15470 & 14298 & 15470 \\
\hline
\end{tabular}

${ }^{*} p<0.10,{ }^{* *} p<0.05,{ }^{* * *} p<0.01$

Notes: t-statistics are reported in parentheses. The standard errors for every regression are clustered at the website-country level. We aggregate every dependent variable to the website-country-browser-OS-product type-week level between weeks 16 and 29 , including both weeks 16 and 29 (April 13th - July 20th). The dependent variable in the regression reported in the first column is the proportion of purchasers associated with each observation and the second column is the average predicted probability. The dependent variables in the third and fourth column are the MSE and AUC, respectively. Finally, in the fifth and sixth columns the dependent variables are the MSE conditional on the true class of the observation.

Table 4 displays the difference-in-differences estimates for all of the relevant prediction related outcome variables. First, column (1) shows that GDPR results in a small but significant

\footnotetext{
${ }^{49}$ We drop observations that either have no purchase data or where the class proportion is degenerate. There are also two websites that we know had a reporting error for purchase data during our sample period and we drop them from our analysis. Further, we drop any (browser, OS, product, website, country) tuple that, on average, has fewer than 50 consumers a week since these observations are very noisy due to low sample sizes and the performance of the prediction problem is less interesting in these cases.
} 
increase in the proportion of purchasers. ${ }^{50}$ Meanwhile, the insignificant coefficient for average prediction probability (i.e. $\hat{p}_{i, j, k}$ ) in column (2) shows little adjustment by the classifier of the firm to this change. Figure 15 in Appendix $G$ displays the time-varying specification for these outcome variables indicating that the average predicted probability remains constant whereas the class proportion fluctuates but appears to increase.

Columns (3) and (4) show the impact of GDPR on the prediction performance of the intermediary as measured in MSE and AUC, respectively. Column (3) shows a significant increase in MSE after GDPR. However, rather than indicating the worsened prediction performance, this is likely to be an artifact of the change in class proportion and the lack of adjustment by the classifier. ${ }^{51}$ Indeed, columns (5) and (6) show that MSE conditional on true class has not gone up; if anything, they have gone down albeit statistically insignificantly. As mentioned above, given the skewed distribution, an increase in the proportion of purchasers will raise the MSE. In fact, column (4) shows a positive estimate for the treatment effect on AUC indicating a marginal improvement in prediction, though it is not statistically significant. The marginal improvement in AUC indicates that the intermediary's ability to separate the two classes has increased. This observation is consistent with what we would expect from the aforementioned increase in consumer persistence.

Finally, Figure 16 in Appendix G displays the results from the time-varying specification for MSE and AUC, indicating that there was an initial increase in MSE followed by an eventual decline. This is consistent with the claim that much of the increase in MSE was a result of the lack of rapid adjustment. Furthermore, the increases in AUC do not occur directly after GDPR but rather also occur gradually.

Overall, our results suggest that GDPR has not negatively impacted the ability to predict consumer behavior and if at all, the sign of the treatment effect suggests the opposite. This is further validated by the exercise in Appendix $\mathrm{H}$ which identifies the expected "long run" changes in prediction performance as a result of the changes to the data observed in section 4 . This exercise shows that an increase in trackability will likely improve prediction performance, whereas the change in the overall size of data as a result of GDPR should not adversely impact prediction performance significantly.

\footnotetext{
${ }^{50}$ To interpret this result it is important to recall that this is the set of purchasers on website $j$ and not the set of purchasers more generally. Thus, linking this back to the earlier discussion, this provides some additional evidence for a weak effect of selective consent in the remaining set of consumers. This is since it implies that the average remaining consumer post-GDPR is more likely to purchase on website $j$.

${ }^{51}$ Appendix F.2 decomposes the change of MSE to accounts for the extent to which the increase may have resulted from the classifier's lack of rapid adjustment to the post-GDPR consumer distribution leading the estimated class probabilities to no longer as closely match the empirical class probabilities.
} 


\section{Conclusion}

In this paper we empirically study the effects of data privacy regulation by exploiting the introduction of GDPR as a natural experiment. We use data from an intermediary that contracts with many online travel agencies worldwide, which allows us to investigate the effect of GDPR on a comprehensive set of outcomes. Our analysis focuses on the stipulation of GDPR that effectively required firms to ask consumers for explicit consent to store and process their data. Despite only partial compliance with this aspect of the regulation, we find a meaningful change in the data collected by firms.

Our results paint a novel and interesting picture of how a consumer's privacy decision- particularly the means by which she protects her privacy-may affect the rest of the economy, including other consumers, and the firms and advertisers relying on consumer data. The strong and effective means of privacy protection made available by laws such as GDPR and the more recent CCPA (California Consumer Privacy Act) should help the privacy-concerned consumers to protect their privacy by eliminating their digital footprints. These consumers are thus clear winners of the laws. What about those consumers who opt in? Their welfare will depend on how their data is used by the firms. If their data is used to target advertising and services to their needs, they too could very well be winners of privacy laws, even if their decision to opt in may not have accounted for the externality. However, if their data is used for extracting consumer surplus, e.g., via personalized pricing, the externalities could harm them.

Regarding firms, we find two effects that partially offset each other. On the one hand, we find that advertising prices increase, which can be explained by the increased trackability of consumers. However, our analysis also shows that smaller advertisers such as ours, who are dependent on third party access, are able to collect less data and conduct less business due to consumer opt-out. This puts them at a disadvantage relative to large companies who rely on their first party ecosystem to collect data. Thus, our paper has broader implications beyond the online travel industry and keyword-based advertising markets. Firms in this industry, as others in the digital economy, increasingly compete with the large technology firms such as Google whose reach ex-

pands across many different online markets and for whom consumers have little choice but to accept data processing. Thus, while our results highlight that increased consent requirements may not be wholly negative, if consumers are similarly using such opt-out capabilities at our estimated rates in other markets (such as behaviorally-targeted advertising markets) then such regulation may put smaller firms at a disadvantage relative to the internet giants. It would be important to study the extent and magnitude of these adverse effects. We believe that these insights and directions for future work are useful for the design of the many proposed regulations in the US and around the world that follow in the footsteps of GDPR. 


\section{References}

Abadie, Alberto, Alexis Diamond, and Jens Hainmueller. 2010. "Synthetic control methods for comparative case studies: Estimating the effect of California's tobacco control program." fournal of the American statistical Association, 105(490): 493-505.

Acemoglu, Daron, Ali Makhdoumi, Azarakhsh Malekian, and Asuman Ozdaglar. 2019. "Too Much Data: Prices and Inefficiencies in Data Markets." National Bureau of Economic Research.

Acquisti, Alessandro, Curtis Taylor, and Liad Wagman. 2016. "The economics of privacy." Journal of Economic Literature, 54(2): 442-92.

Acquisti, Alessandro, Leslie K John, and George Loewenstein. 2013. "What is privacy worth?" The fournal of Legal Studies, 42(2): 249-274.

Aridor, Guy, Yishay Mansour, Aleksandrs Slivkins, and Zhiwei Steven Wu. 2020. “Competing bandits: The perils of exploration under competition." arXiv preprint arXiv:2007.10144.

Athey, Susan, Christian Catalini, and Catherine Tucker. 2017. "The digital privacy paradox: Small money, small costs, small talk." National Bureau of Economic Research.

Bajari, Patrick, Victor Chernozhukov, Ali Hortaçsu, and Junichi Suzuki. 2019. "The impact of big data on firm performance: An empirical investigation.” Vol. 109, 33-37.

Bellemare, Marc F, and Casey J Wichman. 2019. "Elasticities and the inverse hyperbolic sine transformation." Oxford Bulletin of Economics and Statistics.

Berendt, Bettina, Oliver Günther, and Sarah Spiekermann. 2005. "Privacy in e-commerce: stated preferences vs. actual behavior." Communications of the ACM, 48(4): 101-106.

Bergemann, Dirk, Alessandro Bonatti, and Tan Gan. 2019. “The Economics of Social Data."

Boerman, Sophie C, Sanne Kruikemeier, and Frederik J Zuiderveen Borgesius. 2018. "Exploring motivations for online privacy protection behavior: Insights from panel data." Communication Research, 0093650218800915.

Braghieri, Luca. 2019. "Targeted advertising and price discrimination in intermediated online markets." Available at SSRN 3072692.

Buchholz, Nicholas, Laura Doval, Jakub Kastl, Filip Matějka, and Tobias Salz. 2022. "The value of time: Evidence from auctioned cab rides." National Bureau of Economic Research.

Cameron, A Colin, and Pravin K Trivedi. 1990. "Regression-based tests for overdispersion in the Poisson model." fournal of econometrics, 46(3): 347-364.

Cameron, A Colin, and Pravin K Trivedi. 2005. Microeconometrics: methods and applications. Cambridge university press.

Campbell, James, Avi Goldfarb, and Catherine Tucker. 2015. "Privacy regulation and market structure." Journal of Economics \& Management Strategy, 24(1): 47-73. 
Chiou, Lesley, and Catherine Tucker. 2017. "Search engines and data retention: Implications for privacy and antitrust.” National Bureau of Economic Research.

Choi, Jay Pil, Doh-Shin Jeon, and Byung-Cheol Kim. 2019. "Privacy and personal data collection with information externalities." Fournal of Public Economics, 173: 113-124.

Coey, Dominic, and Michael Bailey. 2016. "People and cookies: Imperfect treatment assignment in online experiments.” 1103-1111.

Commission, Data Protection. 2020a. "Guidance Note: Cookies and other tracking technologies."

Commission, Data Protection. 2020b. "Report by the data protection commission on the use of cookies and other tracking technologies.”

Degeling, Martin, Christine Utz, Christopher Lentzsch, Henry Hosseini, Florian Schaub, and Thorsten Holz. 2018. "We Value Your Privacy... Now Take Some Cookies: Measuring the GDPR's Impact on Web Privacy.” arXiv preprint arXiv:1808.05096.

DeGroot, Morris H, and Stephen E Fienberg. 1983. "The comparison and evaluation of forecasters." Journal of the Royal Statistical Society: Series D (The Statistician), 32(1-2): 12-22.

Desmarais, Bruce A, and Jeffrey J Harden. 2013. "Testing for zero inflation in count models: Bias correction for the Vuong test." The Stata fournal, 13(4): 810-835.

Dubé, Jean-Pierre, and Sanjog Misra. 2019. “Personalized pricing and customer welfare.” Available at SSRN 2992257.

Dwork, Cynthia. 2011. "Differential privacy." Encyclopedia of Cryptography and Security, 338340.

Dwork, Cynthia, and Aaron Roth. 2014. “The algorithmic foundations of differential privacy." Foundations and Trends ${ }^{\circledR}$ in Theoretical Computer Science, 9(3-4): 211-407.

Einav, Liran, and Jonathan Levin. 2014. "Economics in the age of big data." Science, 346(6210): 1243089.

Fairfield, Joshua AT, and Christoph Engel. 2015. "Privacy as a public good." Duke Lf, 65: 385.

Fawcett, Tom. 2006. "An introduction to ROC analysis." Pattern recognition letters, 27(8): 861-874.

Godinho de Matos, Miguel, and Idris Adjerid. 2021. "Consumer consent and firm targeting after GDPR: The case of a large telecom provider." Management Science.

Goldberg, Samuel, Garrett Johnson, and Scott Shriver. 2021. "Regulating Privacy Online: An Economic Evaluation of the GDPR.” Available at SSRN 3421731.

Goldfarb, Avi, and Catherine E Tucker. 2011. "Privacy regulation and online advertising." Management science, 57(1): 57-71.

Goldfarb, Avi, and Catherine Tucker. 2012a. "Privacy and innovation." Innovation policy and the economy, 12(1): 65-90.

Goldfarb, Avi, and Catherine Tucker. 2012b. "Shifts in privacy concerns." American Economic 
Review, 102(3): 349-53.

Jia, Jian, Ginger Zhe Jin, and Liad Wagman. 2018. "The short-run effects of GDPR on technology venture investment." National Bureau of Economic Research.

Jia, Jian, Ginger Zhe Jin, and Liad Wagman. 2020. "GDPR and the Localness of Venture Investment.” Available at SSRN 3436535.

Johnson, Garrett. 2013. "The impact of privacy policy on the auction market for online display advertising."

Johnson, Garrett A, Scott K Shriver, and Shaoyin Du. 2020. "Consumer privacy choice in online advertising: Who opts out and at what cost to industry?" Marketing Science.

Johnson, Garrett, Scott Shriver, and Samuel Goldberg. 2020. "Privacy \& market concentration: Intended \& unintended consequences of the GDPR.” Available at SSRN 3477686.

Kehoe, Patrick J, Bradley J Larsen, and Elena Pastorino. 2018. "Dynamic Competition in the Era of Big Data." Working paper, Stanford University and Federal Reserve Bank of Minneapolis.

Lambert, Diane. 1992. "Zero-inflated Poisson regression, with an application to defects in manufacturing." Technometrics, 34(1): 1-14.

Liang, Annie, and Erik Madsen. 2019. "Data Sharing and Incentives." Available at SSRN 3485776.

Lin, Tesary. 2022. "Valuing intrinsic and instrumental preferences for privacy." Marketing Science.

Lin, Tesary, and Sanjog Misra. 2022. "Frontiers: the identity fragmentation bias." Marketing Science.

Norberg, Patricia A, Daniel R Horne, and David A Horne. 2007. "The privacy paradox: Personal information disclosure intentions versus behaviors." fournal of consumer affairs, 41(1): 100-126.

Peukert, Christian, Stefan Bechtold, Michail Batikas, and Tobias Kretschmer. 2022. "Regulatory spillovers and data governance: Evidence from the GDPR.” Marketing Science.

Prince, Jeffrey, and Scott Wallsten. 2020. "How Much is Privacy Worth Around the World and Across Platforms?" Available at SSRN.

Utz, Christine, Martin Degeling, Sascha Fahl, Florian Schaub, and Thorsten Holz. 2019. “(Un) informed Consent: Studying GDPR Consent Notices in the Field.” 973-990, ACM.

Vuong, Quang H. 1989. "Likelihood ratio tests for model selection and non-nested hypotheses." Econometrica: Journal of the Econometric Society, 307-333.

Zhuo, Ran, Bradley Huffaker, Shane Greenstein, et al. 2021. "The impact of the General Data Protection Regulation on internet interconnection." Telecommunications Policy, 45(2): 102083.

Zou, Yixin, Kevin Roundy, Acar Tamersoy, Saurabh Shintre, Johann Roturier, and Florian Schaub. 2020. "Examining the adoption and abandonment of security, privacy, and identity theft protection practices." $1-15$. 


\section{Appendix}

\section{A Additional Consumer Response Figures}

Figure 8: Week by Week Treatment Effect (Cookies and Recorded Searches)
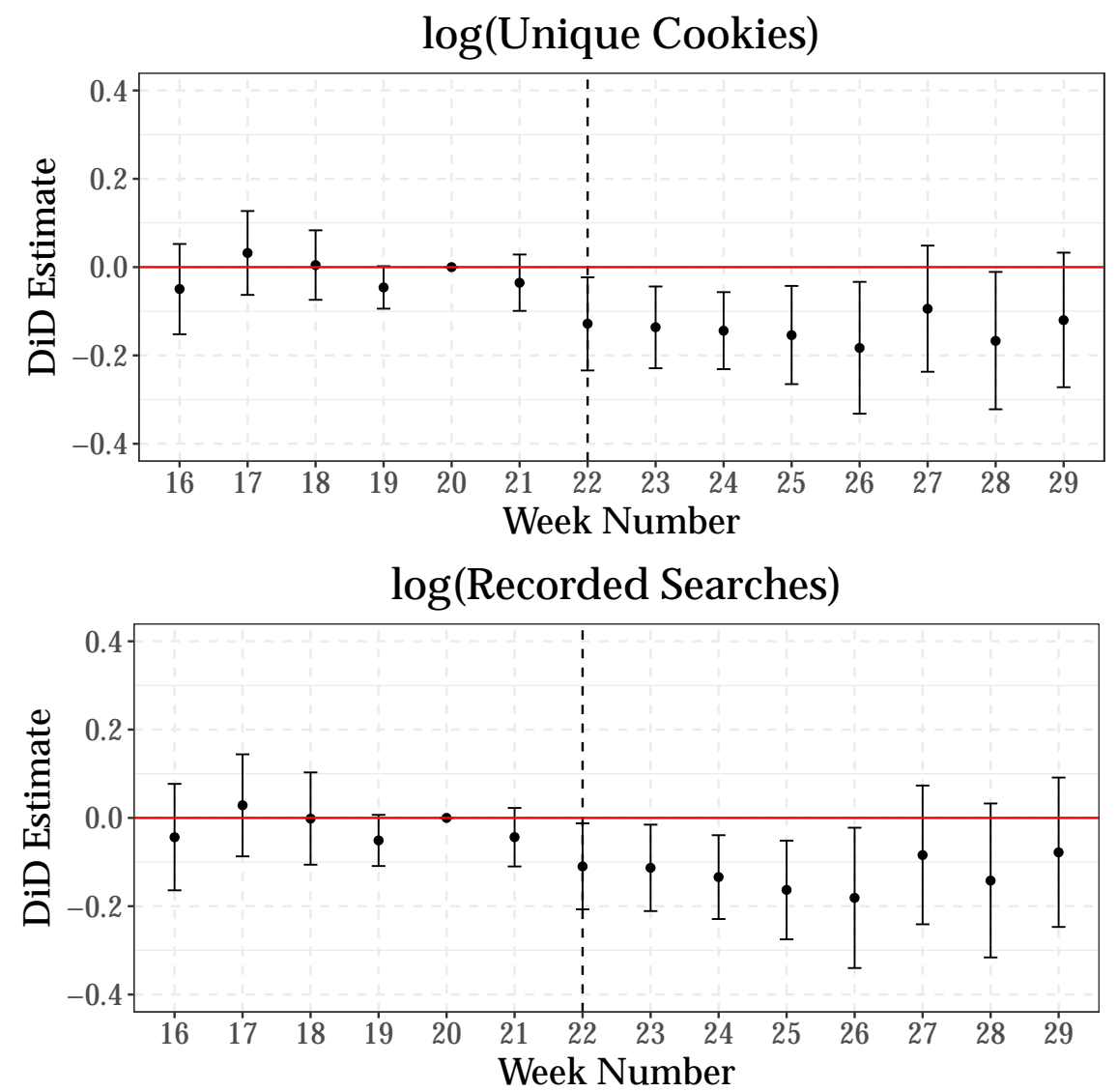

Table 5: Summary Statistics of Consumer Persistence

\begin{tabular}{lcccc}
\hline Region & 1 Week & 2 Weeks & 3 Weeks & 4 Weeks \\
\hline non-EU & .0640 & .0417 & .0330 & .0282 \\
EU & .0962 & .0730 & .0644 & .0597 \\
\hline
\end{tabular}

Notes: The summary statistics are computed on the sample period before GDPR and show the mean consumer persistence values across the $\mathrm{EU}$ and the non-EU for $k=1,2,3,4$. 
Figure 9: Distribution of Consumer Persistence (1 Week)

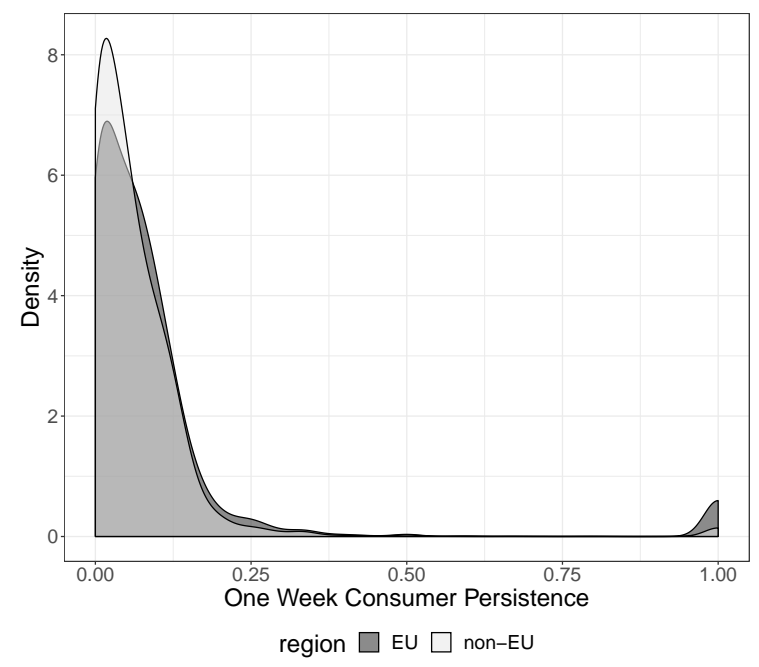

Table 6: Difference-in-Differences Estimates for Sales Activity

(1)

(2)

Total Pages Total Advertising Units

DiD Coefficient

$-0.0387$

0.0837

$(-0.58)$

Product Category Controls

$\checkmark \quad \checkmark$

Week FE

Website $\times$ Country FE
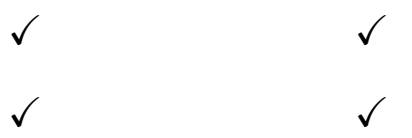

Observations

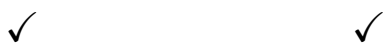

${ }^{*} p<0.10,{ }^{* *} p<0.05,{ }^{* * *} p<0.01$

Notes: t-statistics are reported in parentheses. The standard errors for every regression are clustered at the website-country level. We aggregate every dependent variable to the website-country-product type-week level between weeks 16 and 29, including both weeks 16 and 29 (April 13th - July 20th). The dependent variable in the first regression is the total number of pages where the intermediary is present. The dependent variable in the second regression is the total number of advertising units associated with the intermediary. 
Figure 10: Week by Week Treatment Effect (Consumer Persistence)
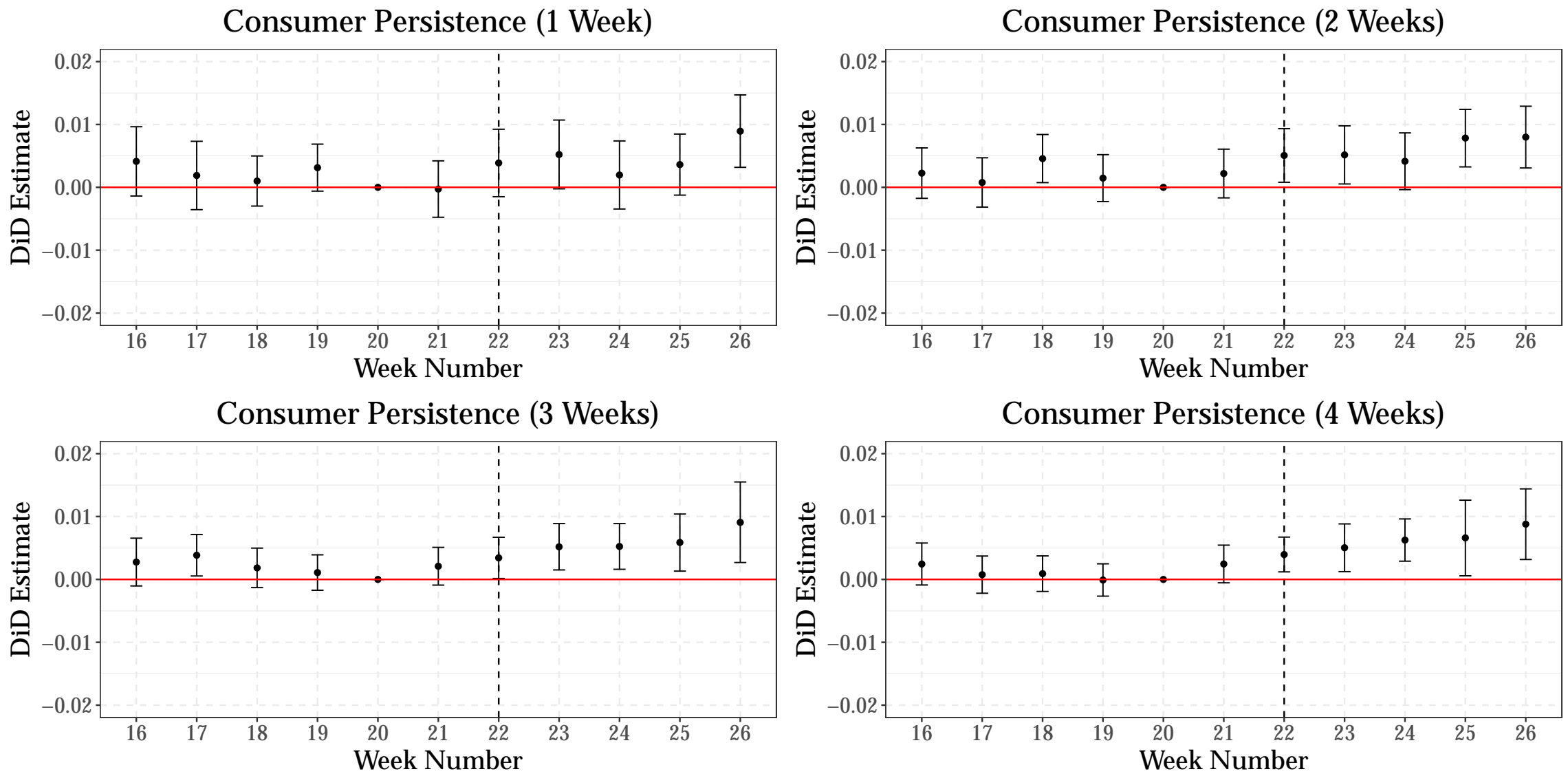
Table 7: Persistence - Large vs. Small Website Heterogeneous Treatment Effects

\begin{tabular}{lcccc}
\hline & $(1)$ & $(2)$ & $(3)$ & $(4)$ \\
& 1 Week & 2 Weeks & 3 Weeks & 4 Weeks \\
& Persistence & Persistence & Persistence & Persistence \\
\hline Treated & 0.000204 & 0.00230 & 0.00183 & $0.00347^{*}$ \\
& $(0.10)$ & $(1.29)$ & $(1.21)$ & $(1.85)$ \\
Large Website $\times$ Treated & 0.00367 & 0.00239 & $0.00255^{*}$ & 0.00202 \\
& $(1.49)$ & $(1.22)$ & $(1.79)$ & $(1.22)$ \\
Product Type Controls & $\checkmark$ & $\checkmark$ & $\checkmark$ & $\checkmark$ \\
OS + Browser Controls & $\checkmark$ & $\checkmark$ & $\checkmark$ & $\checkmark$ \\
Week FE & $\checkmark$ & $\checkmark$ & $\checkmark$ & $\checkmark$ \\
Website $\times$ Country FE & $\checkmark$ & $\checkmark$ & $\checkmark$ & $\checkmark$ \\
\hline Observations & 50160 & 50160 & 50160 & 50160 \\
\hline
\end{tabular}

${ }^{*} p<0.10,{ }^{* *} p<0.05,{ }^{* * *} p<0.01$

Notes: $\mathrm{t}$-statistics are reported in parentheses. The standard errors for every regression are clustered at the website-country level. We aggregate every dependent variable to the website-countrybrowser-OS-product type-week level between weeks 16 and 26, including both weeks 16 and 26 (April 13th - June 29th). The dependent variables in the regression are the consumer persistence measures for $k=1,2,3,4$, respectively. We report heterogeneous treatment effects across large vs. small websites. A website is denoted as large (resp. small) if they are above (resp. below) the median website size where size is defined as average number of weekly searches on the website pre-GDPR. The omitted category is small websites. 


\section{B Robustness for Consumer Response Results}

Figure 11: Synthetic Controls for Cookies and Recorded Searches
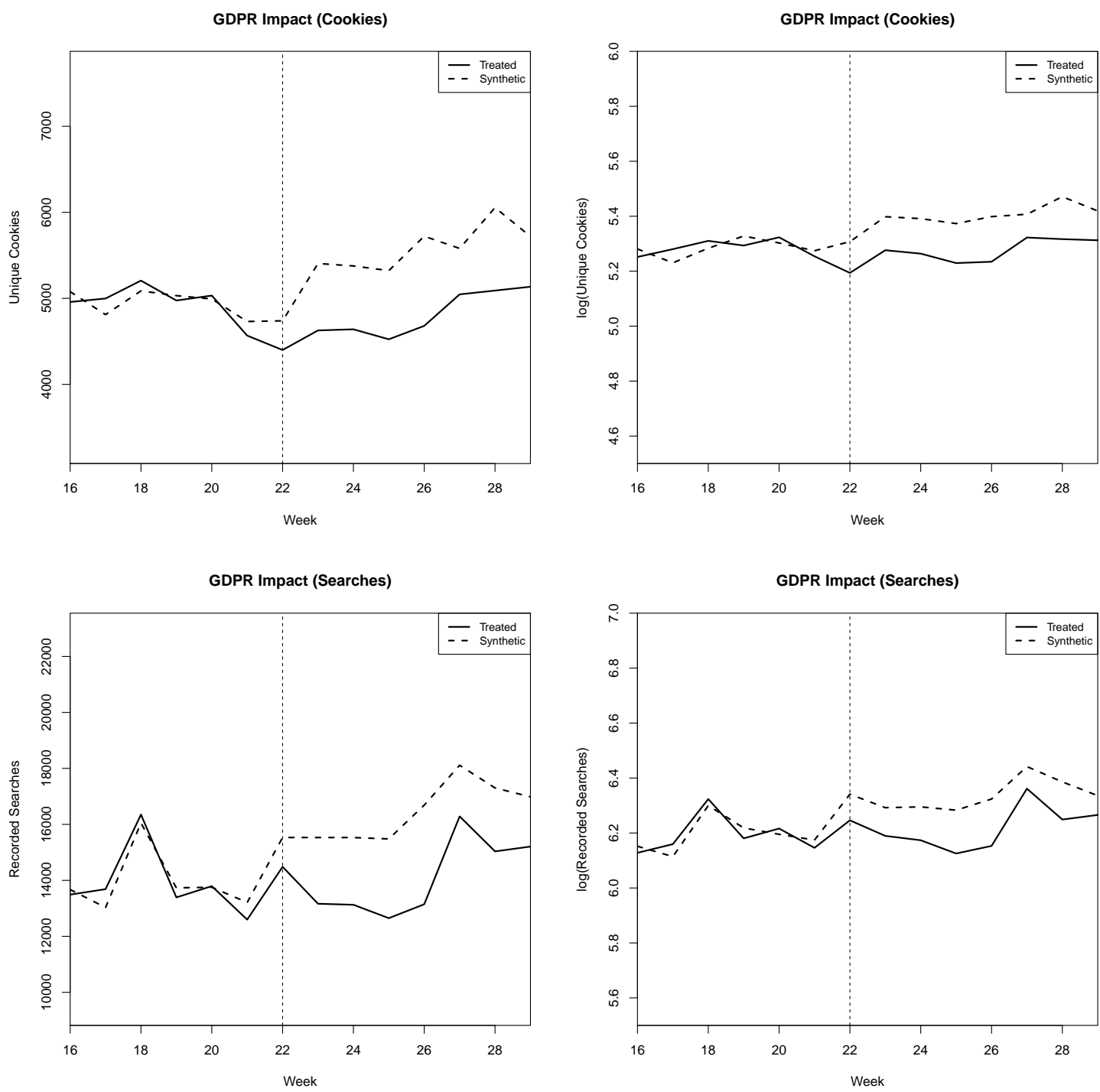

Notes: The plots in the leftmost column display the time series of the average treated unit and the constructed synthetic control for the number of unique cookies (top) and number of recorded searches (bottom). The plots in the rightmost column display the difference at every point in time between the averaged treated unit and the constructed synthetic control for the log of the number of unique cookies (top) and the log of the number of recorded searches (bottom).

We provide additional evidence of robustness for our estimated effects on the usage of consentbased opt-out as a result of GDPR. subsection B.1 mimics the exercise in the main text, but utilizes a standard synthetic control based approach and recovers similar results as our difference-indifferences approach. subsection B.2 augments our analysis with Google Trends data and uses 
this to control for seasonality differences in travel patterns across the countries in our analysis.

\section{B.1 Synthetic Controls}

In order to provide additional validation for the difference-in-differences results in subsection 4.1, we supplement our primary analysis with a synthetic controls analysis, following Abadie, Diamond and Hainmueller (2010) and utilizing the corresponding R package, Synth. We aggregate the data to the same level as we do in the primary analysis. ${ }^{52}$ We expand the set of control countries beyond the United States, Canada, and Russia to include Argentina, Brazil, Australia, Japan, and Mexico in order to allow for additional flexibility in the design of the synthetic control group. ${ }^{53}$ Thus, the travel websites in these countries serve as the possible donor pool for the construction of the synthetic control. In order to apply the synthetic control method to our data, we construct a single average treated unit for each outcome variable from the set of treated units. The set of predictor variables that we utilize in order to fit the weights assigned to each control unit are the two outcome variables that we consider-the total number of searches and the total number of unique cookies observed. We fit the weights to match the outcome variable between weeks 16 and 21. The results of applying this method are reported in Figure 11. Qualitatively, the results match what we find utilizing the difference-in-differences analysis with a stark drop at the onset of GDPR with a small recovery nearing the end of our sample period.

\section{B.2 Controlling for Differences in Travel Patterns Across Countries}

Since our paper tries to understand the impact of privacy regulations utilizing data from the online travel industry, a potential concern is that differential seasonality trends in travel across countries may influence the results. We selected the set of control countries in our analysis specifically to have similar seasonal travel patterns as the major EU countries impacted by GDPR in a short period around the GDPR implementation date. To further validate this we make use of data from Google Trends. The Google Trends data is useful since it provides an estimate of similar quantities observed in our data, but without the possibility that data can be removed as a result of GDPR. We first plot the relative trends over time of a common travel keyword and provide evidence that the travel trends are relatively similar in the period that we study. If anything, such trends result

\footnotetext{
${ }^{52} \mathrm{We}$ also do this exercise aggregating at the website-country level and find qualitatively similar results. One might argue for this aggregation since the way we utilize the synthetic control method involves collapsing all treated units into a single average treated unit and it seems more natural to do so at the website-country level so that the synthetic control represents a synthetic European website. However, this makes the comparison of estimated treatment effects to the primary specification more difficult since the underlying units are different.

${ }^{53}$ Our results are nearly identical if we use the same set of control countries as we do in the baseline difference-indifferences specification, but we use the larger set of countries due to the flexibility of the synthetic control method which makes this a special case of the reported exercise.
} 
Figure 12: Historical Google Trends Travel Patterns

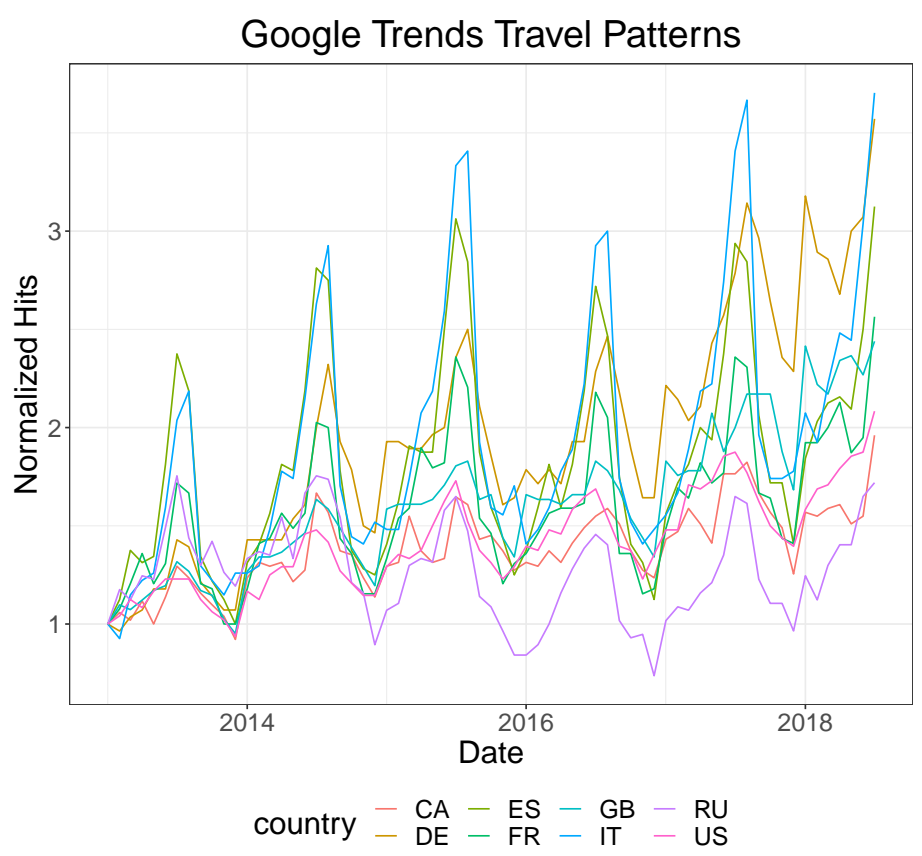

Notes: The graph is constructed by pulling Google Trends data for keyword "booking" for the time period ranging from $1 / 1 / 2013-7 / 31 / 2018$. We pull the data for each country separately. We further normalize the score returned from Google Trends by dividing by the first observation for each country in order to ease cross-country comparisons.

in our estimates understating the treatment effects of GDPR. We then make use of the historical data from Google Trends to better control for seasonal patterns and investigate the impact of these controls on our estimates of the change in total recorded searches and unique cookies.

According to the Google Trends documentation, their data is constructed by a representative sample of searches done through Google Search. Instead of reporting the raw number of searches, Google Trends reports a normalized score that is constructed by dividing the number of searches for the keyword by the total searches of the selected geography and time range. The resulting number is scaled on a range of 0 to 100 based on the topic's proportion to all searches. ${ }^{54}$

Given this data construction, in order to compare the relative intensity of travel queries across countries we pull the data for each country and keyword individually. The first important detail is that Google Trends aggregates across specific strings and not terms, which means that when we do cross country comparisons we have to be careful about the precise keyword we utilize. In order to overcome this difficulty, we use the term of a common and popular OTA across all the countries in our analysis: booking. Figure 12 plots the results from Google Trends for the trends for this keyword from January 1st, 2013 until July 31st, 2018. Figure 12 shows that the keyword appears to pick up the seasonal trends we would expect across the different countries

\footnotetext{
${ }^{54}$ Google Trends Documentation (Retrieved on May 1st, 2022) provides additional details.
} 
as well as that these appear to be similar across this set of countries, especially in the periods of our analysis.

Table 8: Difference-in-Differences Estimates With Google Trends Controls

\begin{tabular}{lcccc}
\hline & $(1)$ & $(2)$ & $(3)$ & $(4)$ \\
& $\begin{array}{c}\log (\text { Unique } \\
\text { Cookies) }\end{array}$ & $\begin{array}{c}\text { Unique } \\
\text { Cookies }\end{array}$ & $\begin{array}{c}\log (\text { Recorded } \\
\text { Searches) }\end{array}$ & $\begin{array}{c}\text { Recorded } \\
\text { Searches }\end{array}$ \\
\hline DiD Coefficient & $-0.129^{* *}$ & $-1373.1^{*}$ & $-0.113^{* *}$ & $-9555.9^{* *}$ \\
& $(-2.52)$ & $(-1.75)$ & $(-1.98)$ & $(-2.25)$ \\
Google Trends Seasonality Controls & $\checkmark$ & $\checkmark$ & $\checkmark$ & $\checkmark$ \\
OS + Browser Controls & $\checkmark$ & $\checkmark$ & $\checkmark$ & $\checkmark$ \\
Product Category Controls & $\checkmark$ & $\checkmark$ & $\checkmark$ & $\checkmark$ \\
Week FE & $\checkmark$ & $\checkmark$ & $\checkmark$ & $\checkmark$ \\
Website $\times$ Country FE & $\checkmark$ & $\checkmark$ & $\checkmark$ & $\checkmark$ \\
\hline Observations & 63840 & 63840 & 63840 & 63840 \\
\hline
\end{tabular}

${ }^{*} p<0.10,{ }^{* *} p<0.05,{ }^{* * *} p<0.01$

Notes: t-statistics are reported in parentheses. The standard errors for every regression are clustered at the website-country level. We aggregate every dependent variable to the website-country-browser-OS-product type-week level between weeks 16 and 26, including both weeks 16 and 26 (April 13th - June 29th). These regressions are identical to those presented in the main text, but with the addition of Google Trends data in order to control for potential differences in seasonal travel patterns across the countries in our analysis.

We now consider the same specification as in (1), but make use of the Google Trends data to additionally construct controls for seasonal travel trends. We run the following regression in order to construct these controls, using the daily Google Trends data from 2013-2018: ${ }^{55}$

$$
\text { google }_{c, t}=\chi[\text { week } \times \text { country }]+\epsilon_{c, t}
$$

where as in the main specification, $t$ denotes week and $c$ denotes country. We then take $\hat{\chi}$ and

\footnotetext{
${ }^{55}$ For this analysis we aggregate the daily Google Trends normalized scores to a weekly level. We define a week in an identical manner as in the primary analysis, from Friday-to-Friday, and take the average normalized score over the week in order to construct this data.
} 
add into our primary specification:

$$
y_{t, c, j, o, b, p}=\alpha_{t}+\hat{\chi}_{c, t}+\delta_{j, c}+\gamma_{o}+\zeta_{b}+\omega_{p}+\beta\left(E U_{j} \times \text { after }_{t}\right)+\epsilon_{t, c, j, o, b, p}
$$

where the notation is identical to that utilized in the main text and $\hat{\chi}_{c, t}$ denotes the coefficient on week $\times$ country that comes from from running (5). The regression results are reported in Table 8 and are quantitatively consistent with the results from our main specification.

\section{Persistence Treatment Effects by Browser/OS}

We further investigate the mechanisms behind the increased consumer persistence by estimating heterogeneous treatment effects across web browsers and operating systems. We exploit the fact that different browsers and operating systems attract different types of individuals with different levels of technical sophistication as well as provide different levels of cookie management.

First, we study heterogeneous treatment effects across web browsers and restrict attention to the most popular web browsers: Google Chrome, Microsoft Edge, Mozilla Firefox, Internet Explorer, Opera, and Apple Safari. We consider the following specification:

$$
y_{t, c, j, o, b, p}=\alpha_{t}+\delta_{j, c}+\gamma_{o}+\zeta_{b}+\omega_{p}+\beta\left(E_{j} \times \text { after }_{t} \times \text { browser }\right)+\epsilon_{t, c, j, o, b, p}
$$

We focus primarily on browsers where it is clear that the two mechanisms should lead to different conclusions. For instance, if we observed similar persistence across Internet Explorer and Google Chrome, this would provide strong evidence for selective consent over privacy means substitution. Internet Explorer is a web browser primarily used on older computers and is known to attract older, less technologically sophisticated users since, at the time of our analysis, it was already phased out by Microsoft for Edge on newer Windows computers. Furthermore, the lack of JavaScript extensions on Internet Explorer makes cookie blockers substantially more difficult to implement on the browser and thus we would expect less "single searchers" and less usage of browser-based privacy means due to the relative lack of automated means of doing so. ${ }^{56}$ We may further expect that different levels of privacy protection among browsers would induce selection in browser choice based on privacy preferences. For instance, Apple Safari and Mozilla Firefox at the time of the GDPR had a broad set of privacy protection means built into it, whereas Google Chrome had laxer privacy controls. ${ }^{57}$ While the selection into web browsers based on privacy preferences, especially during the time period of analysis, is not as clear cut of an indicator, if we

\footnotetext{
${ }^{56}$ See, for instance, Is Ad Block Available on Internet Explorer? Retrieved on January 29th, 2022.

${ }^{57}$ Safari also is the default browser on OS $\mathrm{X}$ and so one would expect users to potentially be less technically sophisticated than those that make use of non-default browsers.
} 
observe little differences between these browsers, then it provides some evidence for selective consent.

Table 10 displays the regression results for this specification with Chrome as the omitted browser. The treatment effect is consistent across browsers with the exception of Internet Explorer which has almost no change in persistence, consistent with privacy means substitution. The estimated treatment effect is lower in Safari relative to Chrome, but the difference is not statistically significant. Both of these observations are consistent with privacy means substitution, though the lack of definitive differences between Chrome and Safari/Firefox does suggest that selective consent may also play a role.

Next, we study heterogeneous treatment effects across operating systems and narrow down the sample to only look at the most popular operating systems: Android, Chrome OS, iOS, Linux, Mac OS X, and Windows. We consider the following specification:

$$
y_{t, c, j, o, b, p}=\alpha_{t}+\delta_{j, c}+\gamma_{o}+\zeta_{b}+\omega_{p}+\beta\left(E U_{j} \times \operatorname{after}_{t} \times O S\right)+\epsilon_{t, c, j, o, b, p}
$$

We are mainly interested in differences in the treatment effects between mobile and desktop consumers. The reason is that there are less readily available privacy means for cookie management on the mobile web compared to desktop and consumer behavior in general tends to be different on mobile compared to desktop. For consistency with privacy means substitution, we would expect a larger difference in persistence on desktop compared to mobile whereas for consistency with selective consent we should expect a smaller difference.

Table 9 displays the regression results with Windows as the omitted operating system that indicates that Android and iOS have no or weak increases in persistence for $k=1,2$ but appear to have an increase in persistence for $k=3,4$. Otherwise, the treatment effect is approximately the same across the different operating systems. Since there seems to be a weak difference between persistence on mobile and desktop this appears to be suggestive of privacy mean substitution, but does not provide conclusive evidence. Overall, the results point to evidence for privacy means substitution, while leaving room for selective consent to play a role in explaining the increase in persistence. 
Table 9: Consumer Persistence - OS Heterogeneous Treatment Effects

\begin{tabular}{|c|c|c|c|c|}
\hline & $\begin{array}{c}(1) \\
1 \text { Week }\end{array}$ & $\begin{array}{c}(2) \\
2 \text { Weeks }\end{array}$ & $\begin{array}{c}(3) \\
3 \text { Weeks }\end{array}$ & $\begin{array}{c}(4) \\
4 \text { Weeks }\end{array}$ \\
\hline Treated & $\begin{array}{c}0.00603^{* * *} \\
(2.70)\end{array}$ & $\begin{array}{c}0.00462^{* * *} \\
(2.76)\end{array}$ & $\begin{array}{c}0.00460^{* * *} \\
(2.65)\end{array}$ & $\begin{array}{c}0.00476^{* * *} \\
(2.91)\end{array}$ \\
\hline Treated $\times(\mathrm{OS}=\mathrm{ANDROID})$ & $\begin{array}{c}-0.00886^{* * *} \\
(-3.19)\end{array}$ & $\begin{array}{c}-0.00429^{*} \\
(-1.96)\end{array}$ & $\begin{array}{c}-0.00256 \\
(-1.26)\end{array}$ & $\begin{array}{c}0.000311 \\
(0.17)\end{array}$ \\
\hline Treated $\times(\mathrm{OS}=$ CHROME_OS $)$ & $\begin{array}{c}-0.00384 \\
(-0.67)\end{array}$ & $\begin{array}{c}-0.00592 \\
(-1.24)\end{array}$ & $\begin{array}{c}-0.00593 \\
(-1.44)\end{array}$ & $\begin{array}{c}0.00176 \\
(0.52)\end{array}$ \\
\hline Treated $\times(\mathrm{OS}=\mathrm{iOS})$ & $\begin{array}{c}-0.00367 \\
(-1.29)\end{array}$ & $\begin{array}{c}-0.00184 \\
(-0.77)\end{array}$ & $\begin{array}{c}0.000438 \\
(0.19)\end{array}$ & $\begin{array}{c}0.00132 \\
(0.70)\end{array}$ \\
\hline Treated $\times(\mathrm{OS}=\mathrm{LINUX})$ & $\begin{array}{c}-0.000856 \\
(-0.18)\end{array}$ & $\begin{array}{c}0.00326 \\
(0.77)\end{array}$ & $\begin{array}{c}-0.000188 \\
(-0.06)\end{array}$ & $\begin{array}{c}0.000463 \\
(0.12)\end{array}$ \\
\hline Treated $\times(\mathrm{OS}=$ MAC_OS_X $)$ & $\begin{array}{c}-0.00291 \\
(-1.08)\end{array}$ & $\begin{array}{c}-0.000367 \\
(-0.19)\end{array}$ & $\begin{array}{c}-0.00209 \\
(-1.26)\end{array}$ & $\begin{array}{c}-0.00184 \\
(-1.10)\end{array}$ \\
\hline OS = ANDROID & $\begin{array}{c}0.0105^{* * *} \\
(3.56)\end{array}$ & $\begin{array}{c}0.00565^{* *} \\
(2.01)\end{array}$ & $\begin{array}{c}0.00335 \\
(1.20)\end{array}$ & $\begin{array}{c}0.00296 \\
(1.18)\end{array}$ \\
\hline OS $=$ CHROME_OS & $\begin{array}{c}0.00307 \\
(0.89)\end{array}$ & $\begin{array}{c}0.00221 \\
(0.59)\end{array}$ & $\begin{array}{c}-0.000749 \\
(-0.27)\end{array}$ & $\begin{array}{c}-0.00117 \\
(-0.45)\end{array}$ \\
\hline $\mathrm{OS}=\mathrm{iOS}$ & $\begin{array}{c}0.00712^{* * *} \\
(2.66)\end{array}$ & $\begin{array}{c}0.000500 \\
(0.22)\end{array}$ & $\begin{array}{c}-0.0000303 \\
(-0.01)\end{array}$ & $\begin{array}{c}-0.0000989 \\
(-0.05)\end{array}$ \\
\hline OS $=$ LINUX & $\begin{array}{c}-0.0164^{* * *} \\
(-4.37)\end{array}$ & $\begin{array}{c}-0.0119^{* * *} \\
(-3.46)\end{array}$ & $\begin{array}{c}-0.0105^{* * *} \\
(-4.17)\end{array}$ & $\begin{array}{c}-0.00732^{* * *} \\
(-2.87)\end{array}$ \\
\hline OS = MAC_OS_X & $\begin{array}{c}-0.000548 \\
(-0.24)\end{array}$ & $\begin{array}{c}-0.00115 \\
(-0.58)\end{array}$ & $\begin{array}{c}-0.00299^{*} \\
(-1.96)\end{array}$ & $\begin{array}{c}-0.00297^{* * *} \\
(-2.68)\end{array}$ \\
\hline Constant & $\begin{array}{c}0.0835^{* * *} \\
(33.88)\end{array}$ & $\begin{array}{c}0.0619^{* * *} \\
(29.13)\end{array}$ & $\begin{array}{c}0.0557^{* * *} \\
(31.75)\end{array}$ & $\begin{array}{c}0.0497^{* * *} \\
(29.66)\end{array}$ \\
\hline Product Type Controls & $\checkmark$ & $\checkmark$ & $\checkmark$ & $\checkmark$ \\
\hline OS $\times$ Week, OS $\times$ EU Controls & $\checkmark$ & $\checkmark$ & $\checkmark$ & $\checkmark$ \\
\hline Week FE & $\checkmark$ & $\checkmark$ & $\checkmark$ & $\checkmark$ \\
\hline Website $\times$ Country FE & $\checkmark$ & $\checkmark$ & $\checkmark$ & $\checkmark$ \\
\hline Browser Controls & $\checkmark$ & $\checkmark$ & $\checkmark$ & $\checkmark$ \\
\hline Observations & 48301 & 48301 & 48301 & 48301 \\
\hline
\end{tabular}

Notes: $t$-statistics are reported in parentheses. The standard errors for every regression are clustered at the website-country level. We aggregate every dependent variable to the website-country-browserOS-product type-week level between weeks 16 and 26, including both weeks 16 and 26 (April 13th June 29th). We restrict focus only to the most popular operating systems. The dependent variables in the regression are the consumer persistence measures for $k=1,2,3,4$, respectively. treated indicates whether the observation is associated with an EU website and past the GDPR implementation date. treated $\times$ os indicates the heterogeneous treatment effect for the specified os. The coefficients on os indicate the estimated values for the $o s$ fixed effect. The held-out operating system is Windows. 
Table 10: Consumer Persistence - Browser Heterogeneous Treatment Effects

\begin{tabular}{|c|c|c|c|c|}
\hline & $\begin{array}{c}(1) \\
1 \text { Week }\end{array}$ & $\begin{array}{c}(2) \\
2 \text { Weeks }\end{array}$ & $\begin{array}{c}(3) \\
3 \text { Weeks }\end{array}$ & $\begin{array}{c}(4) \\
4 \text { Weeks }\end{array}$ \\
\hline Treated & $\begin{array}{c}0.00615^{* * *} \\
(2.99)\end{array}$ & $\begin{array}{c}0.00645^{\text {*** }} \\
(3.51)\end{array}$ & $\begin{array}{c}0.00519^{* * *} \\
(3.29)\end{array}$ & $\begin{array}{c}0.00628^{* * *} \\
(3.49)\end{array}$ \\
\hline Treated $\times($ Browser $=$ EDGE $)$ & $\begin{array}{c}-0.00134 \\
(-0.35)\end{array}$ & $\begin{array}{c}-0.00169 \\
(-0.61)\end{array}$ & $\begin{array}{c}0.00230 \\
(0.74)\end{array}$ & $\begin{array}{c}0.000132 \\
(0.04)\end{array}$ \\
\hline Treated $\times($ Browser $=$ FIREFOX $)$ & $\begin{array}{c}-0.00413 \\
(-1.60)\end{array}$ & $\begin{array}{c}-0.00214 \\
(-0.89)\end{array}$ & $\begin{array}{c}-0.00260 \\
(-1.43)\end{array}$ & $\begin{array}{c}-0.00166 \\
(-0.84)\end{array}$ \\
\hline Treated $\times($ Browser $=\mathrm{IE})$ & $\begin{array}{c}-0.0101^{* *} \\
(-2.53)\end{array}$ & $\begin{array}{c}-0.00838^{* * *} \\
(-2.67)\end{array}$ & $\begin{array}{c}-0.00375 \\
(-1.54)\end{array}$ & $\begin{array}{c}-0.00497^{* *} \\
(-2.03)\end{array}$ \\
\hline Treated $\times($ Browser $=$ OPERA $)$ & $\begin{array}{c}-0.00935^{*} \\
(-1.95)\end{array}$ & $\begin{array}{c}-0.00396 \\
(-0.83)\end{array}$ & $\begin{array}{c}-0.00344 \\
(-0.94)\end{array}$ & $\begin{array}{c}-0.00335 \\
(-0.86)\end{array}$ \\
\hline Treated $\times($ Browser $=$ SAFARI $)$ & $\begin{array}{c}-0.00185 \\
(-0.69)\end{array}$ & $\begin{array}{c}-0.00332 \\
(-1.43)\end{array}$ & $\begin{array}{c}-0.00280 \\
(-1.44)\end{array}$ & $\begin{array}{c}-0.00225 \\
(-1.12)\end{array}$ \\
\hline Browser $=$ EDGE & $\begin{array}{c}0.00125 \\
(0.36)\end{array}$ & $\begin{array}{c}-0.00226 \\
(-0.78)\end{array}$ & $\begin{array}{c}-0.00144 \\
(-0.42)\end{array}$ & $\begin{array}{c}-0.000568 \\
(-0.18)\end{array}$ \\
\hline Browser $=$ FIREFOX & $\begin{array}{c}-0.00503^{* *} \\
(-2.29)\end{array}$ & $\begin{array}{c}-0.00381^{*} \\
(-1.96)\end{array}$ & $\begin{array}{c}-0.00465^{* * *} \\
(-3.13)\end{array}$ & $\begin{array}{c}-0.00409^{* * *} \\
(-2.92)\end{array}$ \\
\hline Browser $=\mathrm{IE}$ & $\begin{array}{c}-0.0164^{* * *} \\
(-6.73)\end{array}$ & $\begin{array}{c}-0.0113^{* * *} \\
(-5.15)\end{array}$ & $\begin{array}{c}-0.00801^{* * *} \\
(-3.29)\end{array}$ & $\begin{array}{c}-0.00764^{* * *} \\
(-4.18)\end{array}$ \\
\hline Browser $=$ OPERA & $\begin{array}{c}-0.00151 \\
(-0.39)\end{array}$ & $\begin{array}{c}-0.00337 \\
(-1.00)\end{array}$ & $\begin{array}{c}-0.00665^{* *} \\
(-2.22)\end{array}$ & $\begin{array}{c}-0.00596^{* *} \\
(-2.15)\end{array}$ \\
\hline Browser $=$ SAFARI & $\begin{array}{c}-0.00315 \\
(-1.22)\end{array}$ & $\begin{array}{c}-0.00229 \\
(-1.06)\end{array}$ & $\begin{array}{c}-0.00309^{*} \\
(-1.80)\end{array}$ & $\begin{array}{c}-0.00211 \\
(-1.20)\end{array}$ \\
\hline Constant & $\begin{array}{c}0.0861^{* * *} \\
(32.30)\end{array}$ & $\begin{array}{c}0.0647^{* * *} \\
(29.30)\end{array}$ & $\begin{array}{c}0.0575^{* * *} \\
(34.48)\end{array}$ & $\begin{array}{c}0.0568^{* * *} \\
(12.53)\end{array}$ \\
\hline Product Type Controls & $\checkmark$ & $\checkmark$ & $\checkmark$ & $\checkmark$ \\
\hline OS $\times$ Week, OS $\times$ EU Controls & $\checkmark$ & $\checkmark$ & $\checkmark$ & $\checkmark$ \\
\hline Week FE & $\checkmark$ & $\checkmark$ & $\checkmark$ & $\checkmark$ \\
\hline Website $\times$ Country FE & $\checkmark$ & $\checkmark$ & $\checkmark$ & $\checkmark$ \\
\hline OS Controls & $\checkmark$ & $\checkmark$ & $\checkmark$ & $\checkmark$ \\
\hline Observations & 40810 & 40810 & 40810 & 40810 \\
\hline
\end{tabular}

${ }^{*} p<0.10,{ }^{* *} p<0.05,{ }^{* * *} p<0.01$

Notes: t-statistics are reported in parentheses. The standard errors for every regression are clustered at the website-country level. We aggregate every dependent variable to the website-country-browserOS-product type-week level between weeks 16 and 26, including both weeks 16 and 26 (April 13th June 29th). We restrict focus only to the most popular web browsers. The dependent variables in the regression are the consumer persistence measures for $k=1,2,3,4$, respectively. treated indicates whether the observation is associated with an EU website and past the GDPR implementation date. treated $\times$ browser indicates the heterogeneous treatment effect for the specified browser. The coefficients on browser indicate the estimated values for the browser fixed effect. The held-out browser is Google Chrome. 


\section{Model of Single Searcher Inflation}

In this section we more formally investigate how the distribution of searches changes after GDPR in order to better understand the mechanisms driving the increase in persistence observed in subsection 4.2. We model the "single searcher" observation from subsection 4.2 as individuals obfuscating their identities using advertising/cookie blockers that generate distinct "excess" single searchers relative to the natural, non-obfuscated, distribution of searches. Our main objective is to understand the following. First, we investigate whether consumers were making use of such privacy means prior to the introduction of GDPR by estimating the fraction of "excess" single searchers. Second, consistent with privacy means substitution, we determine whether the fraction of consumers doing so has decreased after GDPR is introduced. Third, consistent with selective consent, we explore whether the search distribution of the post-GDPR searches has changed so that the remaining consumers search more on the given website.

\section{D.1 Setup and Hypotheses}

We first describe a simple model that motivates our empirical exercise. Suppose that there are two types of consumers - obfuscators $(o)$ and non-obfuscators $(n)$ - and that each type of consumer generates an observed search history length of $k$. Each consumer has a history of length $k \geq 1$. The distribution of observed history length for the obfuscation consumer type $o$ is degenerate with probability mass 1 at $k=1$. For the obfuscation type $o$, we hypothesize that their observed history length is $k=1$ with probability one. For the non-obfuscation consumer type $n$, the probability of observing search history length $k \geq 1$, conditional on observing a consumer, is denoted by $Q(k ; \theta(x))$, where $\theta$ contains the relevant parameters of probability distribution $Q$ and $x$ denotes a set of observable consumer characteristics. In our setting the lowest count is one, which is why we subtract one from each observation to map it into a standard count model. We denote the fraction of consumers that are obfuscators conditional on observable characteristics $x$ as

$$
\pi(x):=\operatorname{Pr}\{\text { obfuscator } \mid x\}
$$

This setup maps into the following observed share of visits $S_{k}$, where $k$ denotes history length:

$$
\begin{aligned}
& S_{1}=\pi(x)+(1-\pi(x)) \cdot Q(1 ; \theta(x)) \\
& S_{k}=(1-\pi(x)) \cdot Q(k ; \theta(x)), \quad \forall k \geq 1
\end{aligned}
$$

We note that given this set-up $\pi$ and $\theta$ are identified and that we can separately estimate $\pi$ and $\theta$ for the pre-GDPR and post-GDPR period, giving us estimates for $\hat{\pi}^{P R E}, \hat{\pi}^{P O S T}, \hat{\theta}^{P R E}, \hat{\theta}^{P O S T}$. Given these estimates, we can compute the mean search history length before and after GDPR 
denoted as $\bar{Q}\left(\hat{\theta}^{P R E}\right)$ and $\bar{Q}\left(\hat{\theta}^{P O S T}\right)$ respectively. Given this setup, our informal hypotheses can be stated as the following null hypotheses:

1. $H_{0}: \overline{\hat{\pi}^{P R E}}=0, H_{a}: \overline{\hat{\pi}^{P R E}} \neq 0$

2. $H_{0}: \overline{\hat{\pi}^{P O S T}}=\overline{\hat{\pi}^{P R E}}, H_{a}: \overline{\hat{\pi}^{P O S T}}<\overline{\hat{\pi}^{P R E}}$

3. $H_{0}: \bar{Q}\left(\hat{\theta}^{P O S T}\right)=\bar{Q}\left(\hat{\theta}^{P R E}\right), H_{a}: \bar{Q}\left(\hat{\theta}^{P O S T}\right)>\bar{Q}\left(\hat{\theta}^{P R E}\right)$

\section{D.2 Data and Estimation}

For this exercise we restrict attention to the same large hotels website shown in Figure 6. We measure how many searches are associated with each identifier observed before and after GDPR is introduced. In total, we observe more than three million unique identifiers.

We allow the parameters of the model to depend on both the web browser and the operating system. Thus, we allow both the arrival rates and the fraction of obfuscators to vary across these dimensions. Next, we parameterize $\pi(x)$ as follows:

$$
\pi(x)=\left[\exp \left(x^{\prime} \gamma\right)\right] /\left[1+\exp \left(x^{\prime} \gamma\right)\right]
$$

where $\gamma$ is a parameter to be estimated. We consider two possible distributional assumptions for $Q$ : a Poisson distribution and a negative binomial distribution, where the latter allows for additional dispersion. For the Poisson distribution we allow the arrival rate $\lambda(x)$ to vary across observables and we do similarly for the negative binomial parameters $\mu(x), \alpha(x) .{ }^{58}$

Our setup maps almost directly to standard zero-inflation Poisson models (e.g. Lambert (1992). We follow Lambert (1992); Cameron and Trivedi (2005) and estimate the parameters of the model via maximum likelihood estimation. The model with a positive share of obfuscators is tested against either a standard Poisson regression or a negative binomial regression. We then conduct a Vuong test to evaluate whether a model with type $o$ consumers leads to a better fit to the observed data (Vuong, 1989; Desmarais and Harden, 2013). In order to test our second and third hypotheses of interest, we do a t-test comparing the vectors of $\hat{\pi}^{P R E}$ and $\hat{\pi}^{P O S T}$ as well as $Q\left(\hat{\theta}^{P R E}\right)$ and $Q\left(\hat{\theta}^{P O S T}\right)$ respectively.

\section{D.3 Results}

We first consider the specification where we assume that $Q$ follows a Poisson distribution. The results of the Vuong test strongly conclude that there is evidence for the existence of type $O$

\footnotetext{
${ }^{58}$ See section 20.4.1 of Cameron and Trivedi (2005) for full details of the parameterization for Poisson and Negative Binomial regressions that we utilize.
} 
consumers in both periods with a z-statistic of -244.85 in the pre-GDPR period and -246.28 in the post-GDPR period.

We then compare the resulting $\hat{\pi}$ in the pre-GDPR and post-GDPR periods, denoted by $\hat{\pi}^{P R E}$ and $\hat{\pi}^{P O S T}$, respectively. We run a t-test with the null hypothesis that $\overline{\hat{\pi}^{P O S T}} \geq \overline{\hat{\pi}^{P R E}}$. We are able to reject the null with $p<0.001$. The difference is also economically significant as we note that $\overline{\hat{\pi}^{P R E}}=0.478$ and $\overline{\hat{\pi}^{P O S T}}=0.354$, suggesting a significant drop of obfuscators after GDPR. Furthermore, we compare the resulting estimates of $Q\left(\hat{\theta}^{P R E}\right)$ and $Q\left(\hat{\theta}^{P O S T}\right)$ and run a t-test with the null hypothesis that $\bar{Q}\left(\hat{\theta}^{P O S T}\right)=\bar{Q}\left(\hat{\theta}^{P R E}\right)$. We are able to reject the null with $p<0.001$ and with a mean increase of nearly one search per consumer as $\bar{Q}\left(\hat{\theta}^{P R E}\right)=4.37$ and $\bar{Q}\left(\hat{\theta}^{P R E}\right)=5.30$. Thus, this provides evidence that both the fraction of obfuscators fell, but also that the non-obfuscators search more.

One concern with the parameterization of $Q$ as Poisson is that it does not account for overdispersion or underdispersion. We can directly test for overdispersion. Let $Y_{i}$ denote the observed history length for consumer $i$ and $\hat{\lambda}_{i}$ the implied variance of the Poisson distribution. One can then test the null that $\alpha=0$ for $\operatorname{VAR}\left(Y_{i}\right)=\hat{\lambda}_{i}+\alpha \cdot \hat{\lambda}_{i}$ against the alternative that $\alpha$ is larger than zero (Cameron and Trivedi, 1990). We reject the null $(p<0.001)$ in both the pre and post period. Thus, we conclude that the data is overdispersed and consider the common remedy that imposes that $Q$ follows a negative binomial distribution, instead of a Poisson distribution (Cameron and Trivedi, 2005).

Under the the assumption of a negative binomial, the Vuong test still concludes that there is evidence for the presence of type $o$ consumers $(z=-6.97)$ in the pre-GDPR period. However, we no longer reject the model without excess single searchers in the post-GDPR period $(z=-1.81$, AIC-corrected: $z=-0.84$, BIC-corrected: $z=4.94)$. Furthermore, we are able again to reject the null hypothesis that $\hat{\pi}^{P O S T}=\hat{\pi}^{P R E}$ and $\bar{Q}\left(\hat{\theta}^{P O S T}\right)=\bar{Q}\left(\hat{\theta}^{P R E}\right)$ with $p<0.001$.

In sum, we document statistical evidence for obfuscators in the pre-GDPR period under both distributional assumptions. Once we take into account the overdispersion relative to a Poisson count model, we do not find evidence for excess single searchers in the post-GDPR period. Finally, under both distributional assumptions, we find that the mean of the non-obfuscator search history length distribution has increased post-GDPR. 


\section{E Additional Advertisement and Auction Figures}

Table 11: Difference-in-Differences Estimates for Advertisements Delivered

\begin{tabular}{|c|c|c|}
\hline & $\begin{array}{c}(1) \\
\text { Total } \\
\text { Advertisements Delivered }\end{array}$ & $\begin{array}{c}(2) \\
\text { asinh(Total } \\
\text { Advertisements Delivered) }\end{array}$ \\
\hline DiD Coefficient & $\begin{array}{c}-2627.2 \\
(-1.61)\end{array}$ & $\begin{array}{l}-0.145 \\
(-1.52)\end{array}$ \\
\hline OS + Browser Controls & $\checkmark$ & $\checkmark$ \\
\hline Product Category Controls & $\checkmark$ & $\checkmark$ \\
\hline Week FE & $\checkmark$ & $\checkmark$ \\
\hline Website $\times$ Country FE & $\checkmark$ & $\checkmark$ \\
\hline Observations & 62328 & 62328 \\
\hline
\end{tabular}

${ }^{*} p<0.10,{ }^{* *} p<0.05,{ }^{* * *} p<0.01$

Notes: $\mathrm{t}$-statistics are reported in parentheses. The standard errors for every regression are clustered at the website-country level. We aggregate every dependent variable to the website-country-browserOS-product type-week level between weeks 16 and 29, including both weeks 16 and 29 (April 13th July 20th). The dependent variables are the log and overall level of total advertisements delivered to consumers.

Figure 13: Week by Week Treatment Effect (Total Advertisements Delivered)

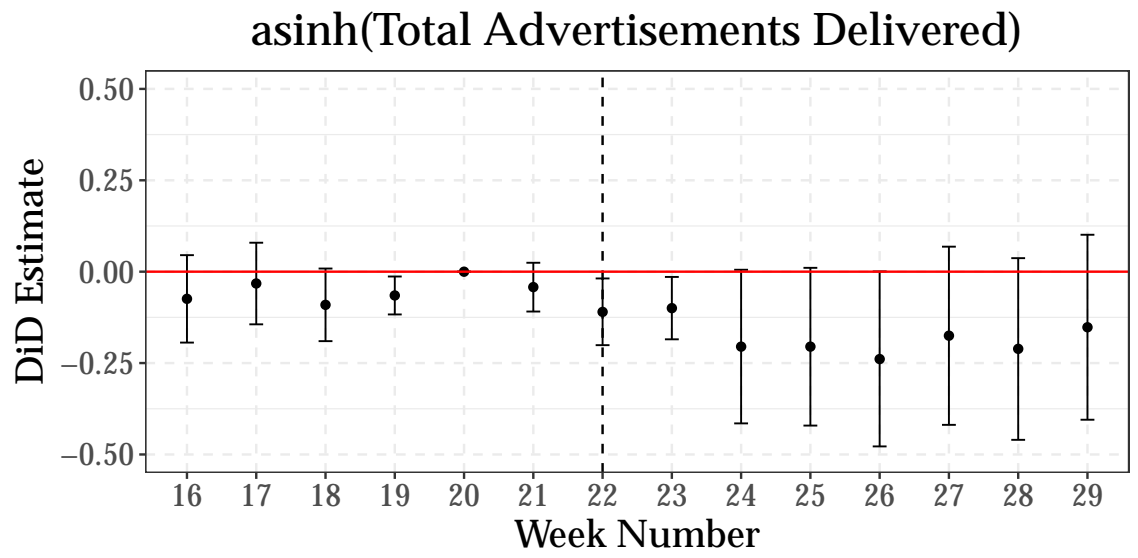


Table 12: Difference-in-Differences Estimates for Fraction of Targeted Advertisements

\begin{tabular}{|c|c|c|c|}
\hline & $\begin{array}{c}\text { (1) } \\
\text { Fraction Exposed to Ads } \\
\text { Overall }\end{array}$ & $\begin{array}{l}(2) \\
\text { Fraction Exposed to Ads } \\
\text { Multi-searchers }\end{array}$ & $\begin{array}{l}(2) \\
\text { Fraction Exposed to Ads } \\
\text { Single-searchers }\end{array}$ \\
\hline DiD Coefficient & $\begin{array}{l}0.0176^{* *} \\
(2.06)\end{array}$ & $\begin{array}{l}0.0242^{* * *} \\
(3.09)\end{array}$ & $\begin{array}{c}0.0165^{*} \\
(1.73)\end{array}$ \\
\hline $\begin{array}{l}\text { OS + Browser Controls } \\
\text { Week FE }\end{array}$ & $\checkmark$ & $\checkmark$ & $\checkmark$ \\
\hline Website $\times$ Country FE & $\checkmark$ & $\checkmark$ & $\checkmark$ \\
\hline Observations & 3602 & 3602 & 3602 \\
\hline \multicolumn{4}{|c|}{${ }^{*} p<0.10,{ }^{* *} p<0.05,{ }^{* * *} p<0.01$} \\
\hline \multicolumn{4}{|c|}{$\begin{array}{l}\text { Notes: t-statistics are reported in parentheses. The standard errors for every regression are clustered at the website-country } \\
\text { level. We aggregate every dependent variable to the website-country-browser-OS-period level between April 13th and July } \\
\text { 20th. The period in the aggregation denotes pre vs. post GDPR. We aggregate to the period level in order to have a sufficiently } \\
\text { long period to track if someone was ever exposed to an advertisement and is a single-searcher. The dependent variable in } \\
\text { column (1) is the fraction of observed cookies that were ever targeted for advertisements. The dependent variable in column } \\
\text { (2) is the fraction of observed multi-searcher cookies that were ever targeted for advertisements. The dependent variable in } \\
\text { column (3) is the fraction of observed single-searcher cookies that were ever targeted for advertisements. }\end{array}$} \\
\hline
\end{tabular}

Table 13: Overall Single vs. Multi Searcher Flight Search Patterns

\begin{tabular}{cccc}
\hline \multicolumn{2}{c}{ Days Before Departure } & \multicolumn{2}{c}{ Fraction of Domestic Flights } \\
Single-Searcher & Multi-Searcher & Single-Searcher & Multi-Searcher \\
\hline $\mathbf{5 7 . 5}$ & 58.0 & 0.305 & 0.297 \\
\hline
\end{tabular}

Notes: The first two columns show the mean number of days before departure for single-searchers and multi-searchers. The final two columns show the mean fraction of domestic flights for single-searchers and multi-searchers. The statistics are computed across the dataset of the website-country-browser-OS-period level aggregation between April 13th - July 20th. The period in the aggregation denotes pre vs. post GDPR.

Table 14: Single vs. Multi Searcher Flight Search Patterns by Region

\begin{tabular}{lcc}
\hline Region & Days Before Departure & Fraction of Domestic Searches \\
\hline \multirow{2}{*}{ non-EU } & $\begin{array}{c}\text { Multi-searchers: } 58.4(0.57) \\
\text { Single-searchers: } 58.1(0.39)\end{array}$ & Multi-searchers: $0.477(.005)$ \\
& Single-searchers: $0.474(.005)$ \\
\hline \multirow{2}{*}{ EU } & Multi-searchers: $57.7(0.58)$ & Multi-searchers: $0.145(0.002)$ \\
& Single-searchers: $56.9(0.49)$ & Single-searchers: $0.165(0.003)$ \\
\hline
\end{tabular}

Notes: The table displays the fraction of searches that are for domestic flights before and after the GDPR across EU vs. non-EU countries. The table separately displays the fraction for multi-searchers - cookies associated with more than one search -and single-searchers - cookies associated with only one search - across EU and non-EU countries. Standard errors are in parentheses. 
Table 15: Mean Market Concentration

\begin{tabular}{lrrrr}
\hline Region & CR-1 & CR-3 & CR-5 & HHI \\
\hline Non-EU & .0744 & .216 & .340 & .0546 \\
EU & .0689 & .204 & .331 & .0542 \\
\hline
\end{tabular}

Notes: The table reports the means of several market concentration measures in the pre-GDPR period for both the EU and the non-EU. The first three columns display the mean market share concentrations of the top 1 , top 3 , and top 5 advertisers according to share of advertisements delivered to consumers (concentration ratio 1,3 , and 5 respectively). The fourth column displays the mean Herfindahl-Hirschman Index (HHI) using the same market share definition.

Table 16: Difference-in-Differences Estimates for Composition of Advertisers

\begin{tabular}{lccccc}
\hline & $(1)$ & $(2)$ & $(3)$ & $(4)$ & $(5)$ \\
& CR-1 & CR-3 & CR-5 & HHI & Mean Number of Bidders \\
\hline DiD Coefficient & $0.00233^{*}$ & $0.00826^{* *}$ & $0.0138^{* *}$ & $0.00227^{*}$ & $-.363^{* * *}$ \\
OS + Browser Controls & $(1.78)$ & $(2.17)$ & $(2.24)$ & $(1.95)$ & $(-4.38)$ \\
Product Category Controls & $\checkmark$ & $\checkmark$ & $\checkmark$ & $\checkmark$ & $\checkmark$ \\
Week FE & $\checkmark$ & $\checkmark$ & $\checkmark$ & $\checkmark$ & $\checkmark$ \\
Website $\times$ Country FE & $\checkmark$ & $\checkmark$ & $\checkmark$ & $\checkmark$ & $\checkmark$ \\
\hline Observations & 62328 & 62328 & 62328 & 62328 & $\checkmark$ \\
\hline$* p<0.10,{ }^{* *} p<0.05,{ }^{* * *} p<0.01$ & & & & $\checkmark 2328$
\end{tabular}

Notes: t-statistics are reported in parentheses. The standard errors for every regression are clustered at the websitecountry level. We aggregate every dependent variable to the website-country-browser-OS-product type-week level between weeks 16 and 29, including both weeks 16 and 29 (April 13th -July 20th). The dependent variables in the regressions reported in the first three columns are the market share concentrations of the top 1, top 3, and top 5 advertisers according to share of advertisements delivered to consumers (concentration ratio 1,3 , and 5 respectively). The dependent variable in the fourth column is the Herfindahl-Hirschman Index (HHI) using the same market share definition. The dependent variable in the fifth column is the mean number of bidders in the auction. We define $C R-I_{j t}$ as the concentration of impressions for the top $I$ out of $K$ advertisers on website $j$ at time $t$. Let $i m p_{k j t}$ be the impressions of the $k$-th largest advertiser (according to impression share) on website $j$ at time $t$. Then, $C R-I_{j t}=\frac{\sum_{k=1}^{I} i m p_{k j t}}{\sum_{k=1}^{K} i m p_{k j t}}$. For a website $j$ and time $t$, the share of advertiser $k \in\{1,2, \ldots, K\}$ is denoted by $s_{k}$. HHI is therefore defined as follows: $H H I_{j t}=\sum_{k=1}^{K} s_{k}^{2}$. 


\section{F Prediction Evaluation Measures}

\section{F.1 AUC Primer}

In this section we provide additional details on how to calculate the AUC measure and its interpretation. To begin, fix the classification threshold at any $\hat{P}$. Then, a consumer with score $\hat{p}_{i, j, k}$ is classified as a purchaser if $\hat{p}_{i, j, k}>\hat{P}$ and a non-purchaser if $\hat{p}_{i, j, k} \leq \hat{P}$. This would result in a false positive rate-a rate at which a non-purchaser is misclassified into a purchaser:

$$
F P R:=\frac{\text { False Positive }}{\text { True Negative + False Positive }}=\frac{\sum_{i, j, k}\left|\left\{\hat{p}_{i, j, k}>\hat{P}, y_{i, j, k}^{T R U E}=0\right\}\right|}{\sum_{i, j, k}\left|\left\{y_{i, j, k}^{T R E}=0\right\}\right|} .
$$

At the same time, it would result in a true positive rate-or a rate at which a purchaser is correctly classified as a purchaser:

$$
T P R:=\frac{\text { True Positive }}{\text { True Positive + False Negative }}=\frac{\sum_{i, j, k}\left|\left\{\hat{p}_{i, j, k}>\hat{P}, y_{i, j, k}^{T R U E}=1\right\}\right|}{\sum_{i, j, k}\left|\left\{y_{i, j, k}^{T R U E}=1\right\}\right|} .
$$

The ROC then depicts the level of TPR a prediction machine achieves for each level of $F P R$ it tolerates.

Figure 14: Sample ROC Curve

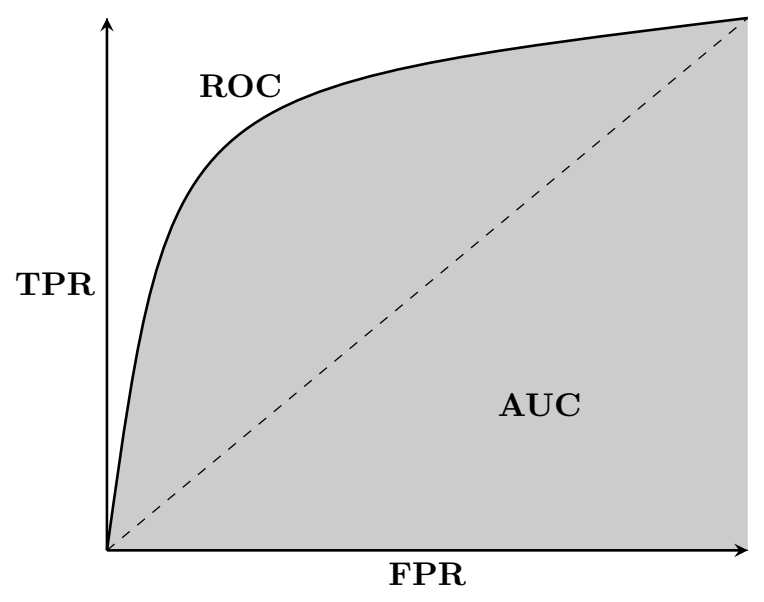

Notes: This figure depicts an ROC curve, which maps out the trade-off between type I and type II errors for a classifier as the classification threshold varies. The area under the ROC curve is denoted by AUC and provides a scalar measure of prediction performance.

The ROC is obtained by tracing the locus of $(F P R, T P R)$ by varying the classification thresh- 
old $\hat{P}{ }^{59}$ The slope of the ROC corresponds to the additional power (in rate) the prediction gains for an additional unit of type I error (in rate) it tolerates. For a random predictor, this slope would be one, and the ROC will be a 45 degrees line. A better than random predictor would produce an ROC which lies above that 45 degrees line. Figure 14 depicts a typical ROC curve.

\section{F.2 Breakdown of MSE}

In this section we further investigate the cause of the increase in MSE in our difference-indifferences analysis in section 6. In order to do so we utilize a standard decomposition for the MSE in the classification context and study the effects of GDPR on each component of the decomposition. The MSE for binary classification problems can be decomposed into a calibration and refinement component (DeGroot and Fienberg, 1983). The calibration component indicates the degree to which the estimated probabilities match the true class proportion. The refinement component indicates the usefulness of the prediction where a more refined prediction is one that is closer to certainty (i.e. closer to 0 or 1 with 0.5 being the most uncertain). Thus, a classifier with a good MSE is well-calibrated and more refined. This decomposition requires a discretization of the estimated probabilities into a series of $K$ bins. ${ }^{60}$ For notation, $p_{k}$ denotes the $k$ th estimated probability bin, $n_{k}$ denotes the number of probability estimates falling into the $k$ th bin and $\bar{o}_{k}$ denotes the true class proportion in the $k$ th bin in the data. This allows us to rewrite (4) as:

$$
M S E_{j}=\underbrace{\frac{1}{\sum_{i \in \mathcal{I}_{j}}\left|\mathcal{K}_{i, j}\right|} \sum_{k=1}^{K} n_{k}\left(p_{k}-\bar{o}_{k}\right)^{2}}_{\text {calibration error }}+\underbrace{\frac{1}{\sum_{i \in \mathcal{I}_{j}}\left|\mathcal{K}_{i, j}\right|} \sum_{k=1}^{K} n_{k} \bar{o}_{k}\left(1-\bar{o}_{k}\right)}_{\text {refinement error }}
$$

We run the same specification utilizing each component of the decomposition of the MSE as the outcome variable. These results are reported in Table 17. They indicate that both the refinement and calibration components increased after GDPR. Both of the components are approximately equally responsible for the increase in MSE with the calibration component being only slightly larger. The increase in calibration error is driven by the classifier's lack of rapid adjustment to the post-GDPR consumer distribution leading the estimated class probabilities to no longer as closely match the empirical class probabilities. However, the increase in refinement error points to a partial adjustment since this increase is a result of the increased uncertainty in the predicted class (i.e. the class proportion moving closer to 0.5).

\footnotetext{
${ }^{59}$ For extreme cases, with $\hat{P}=1$, all consumers are classified as non-purchasers, which yields $(F P R, T P R)=$ $(0,0)$, and with $\hat{P}=0$ all consumers are classified as purchasers, which yields $(F P R, T P R)=(1,1)$.

${ }^{60}$ When calculating the decomposed MSE we will primarily utilize equally spaced bins of size 0.01 . Note that since the decomposition requires this discretization, the decomposed MSE and the standard MSE are not precisely the same quantities, but are approximately the same.
} 
Table 17: Difference-in-Differences Estimates for Relevance and Calibration

\begin{tabular}{lcc}
\hline & $(1)$ & $(2)$ \\
& Calibration & Refinement \\
\hline DiD Coefficient & $0.00735^{* * *}$ & $0.00576^{* *}$ \\
& $(2.84)$ & $(2.64)$ \\
OS + Browser Controls & $\checkmark$ & $\checkmark$ \\
Product Category Controls & $\checkmark$ & $\checkmark$ \\
Week FE & $\checkmark$ & $\checkmark$ \\
Website $\times$ Country FE & $\checkmark$ & $\checkmark$ \\
\hline Observations & 15470 & 15470 \\
\hline
\end{tabular}

${ }^{*} p<0.10,{ }^{* *} p<0.05,{ }^{* * *} p<0.01$

Notes: t-statistics are reported in parentheses. The standard errors for every regression are clustered at the website-country level. We aggregate every dependent variable to the website-countrybrowser-OS-product type-week level between weeks 16 and 29, including both weeks 16 and 29 (April 13th - July 20th). The dependent variable in the regression reported in the first column is the calibration component of the MSE. The dependent variable in the regression reported in the second column is the refinement component of the MSE. 


\section{G Additional Prediction Figures}

Figure 15: Week by Week Treatment Effect (Average Predicted Probability and Class Proportion)
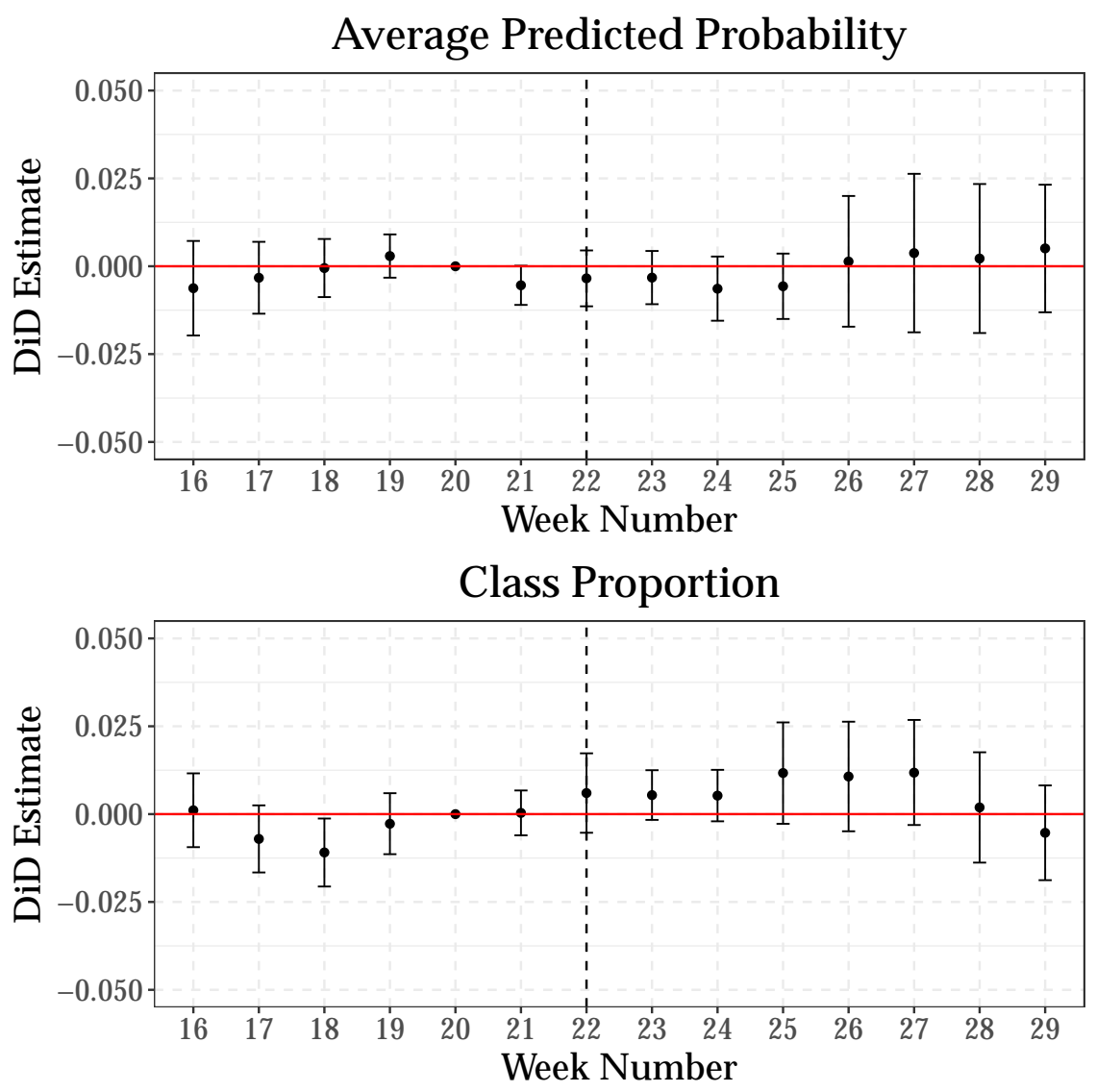
Figure 16: Week by Week Treatment Effect (MSE and AUC)
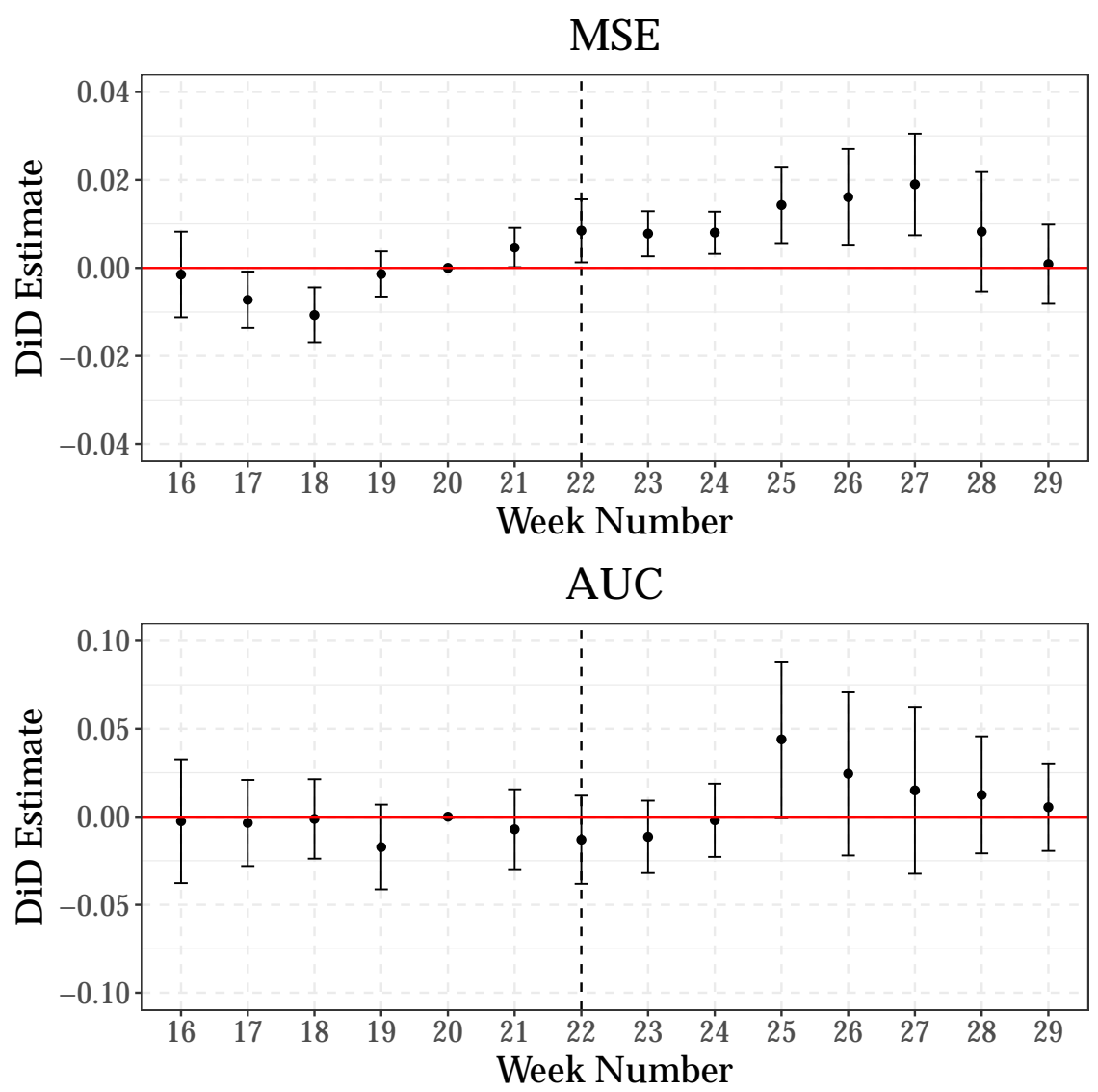

\section{H The Impact of Consumer Persistence and Data Scale on Prediction}

The analysis in section 6 on the effect of GDPR on the firm's ability to predict is limited by the data restrictions and the apparent lack of adjustment by its prediction algorithm to the postGDPR environment. To fully understand the implications for prediction, therefore, we now take a different approach. Instead of asking how the firm's prediction was actually impacted in the immediate aftermath, we now ask what would happen to predictive performance in the long run when the algorithm were fully adjusted.

As observed in section 4, GDPR reduces the number of consumers that the intermediary observes but remaining consumers are more persistently trackable. Our approach is to study how these two features-number of observed consumers and the persistence of observed consumersimpact the two measures of prediction performance cross-sectionally by comparing across web- 
sites differing in these two dimensions. We use a dataset aggregated at the website-product typeweek level. We restrict attention to the pre-GDPR period between January 19th and April 6th. We rely again on the fact that the intermediary only utilizes the data from each individual website in order to train the model for that website. This ensures that predictions for each website are only responsive to the data size and persistence of that website.

We run the following regressions where the dependent variable, $\operatorname{pred}_{t, c, j, p}$ represents the prediction error of website $j$ in country $c$ for product type $p$ at time $t$. The fixed effects are the same as in the primary empirical specification and the standard errors are clustered at the websitecountry level, the same as with the previous specifications:

$$
\begin{aligned}
& \operatorname{pred}_{t, c, j, p}=\beta \cdot \log (\text { Recorded_Searches })+\alpha_{t}+\delta_{j, c}+\omega_{p}+\epsilon_{t, c, j, p} \\
& \text { pred }_{t, c, j, p}=\beta \cdot \text { Consumer_Persistence }+\alpha_{t}+\delta_{j, c}+\omega_{p}+\epsilon_{t, c, j, p}
\end{aligned}
$$

Table 19 displays the OLS estimates of the regression relating total recorded searches on prediction error, using both the MSE and AUC as the dependent variables. We report the results of running the regressions with and without the website and website-country fixed effects, but our preferred specification is the one without the website and website-country fixed effects. ${ }^{61}$ This corresponds to the regression results in Columns (1) and (3) of Table 19. As expected, an increase in the total recorded searches increases AUC significantly and decreases MSE, albeit insignificantly. Recall that our point estimate of the magnitude of lost data from the GDPR was $10.7 \%$. With this data loss, the magnitude of the predicted decline in prediction error is relatively small with a $10.7 \%$ decrease in recorded searches only leading to a 0.0007 decrease in AUC. ${ }^{62}$

Table 18 displays the OLS estimates of the regression relating four week consumer persistence to prediction error, using both the MSE and AUC as the dependent variable. As before, we have regressions with and without website and website-country fixed effects, and focus primarily on the regressions without them. Recall that we previously found a 0.00505 increase in the four week persistence as a result of GDPR. Combined with the point estimates from Table 18, this implies an increase of 0.013 for AUC and a decrease of 0.007 for MSE.

Putting these two results together point to the fact that the decline in the overall scale of data should have little impact on predictability, but the increase in consumer persistence should marginally improve prediction according to both AUC and MSE. However, this does not imply that the scale of data is unimportant which would run counter to standard statistical intuition;

\footnotetext{
${ }^{61}$ The reason is that the website-country fixed effects soak up the variation in different dataset sizes across websites, even though understanding how this variation impacts prediction error is our main interest.

${ }^{62}$ In reality the intermediary does not train its models only on data from the current week, but rather utilizing a sliding window of data that includes previous weeks. Table 20 shows the results for the same specification, but uses a sliding window total of recorded searches instead of the weekly total number of recorded searches, and shows that the point estimates do not change much when taking this into account.
} 
on the contrary, prediction ability improves substantially as the scale of data increases. Rather, the change in the scale of the data as a result of GDPR is not large enough to cause meaningful changes in prediction error in the long run. However, the increase in persistence as a result of GDPR should lead to an improvement in prediction capabilities in the long run.

Table 18: Consumer Persistence and Prediction Error

\begin{tabular}{lcccc}
\hline & $(1)$ & $(2)$ & $(3)$ & $(4)$ \\
& AUC & AUC & MSE & MSE \\
\hline Four Week Persistence & $2.621^{* * *}$ & 0.758 & $-1.401^{* *}$ & $0.611^{*}$ \\
& $(4.55)$ & $(0.95)$ & $(-2.58)$ & $(1.67)$ \\
Constant & $0.542^{* * *}$ & $0.686^{* * *}$ & $0.221^{* * *}$ & $0.0852^{* * *}$ \\
& $(11.35)$ & $(20.17)$ & $(4.91)$ & $(5.30)$ \\
Product Category Controls & $\checkmark$ & $\checkmark$ & $\checkmark$ & $\checkmark$ \\
Week FE & $\checkmark$ & $\checkmark$ & $\checkmark$ & $\checkmark$ \\
Country FE & $\checkmark$ & $\checkmark$ & $\checkmark$ & $\checkmark$ \\
Website $\times$ Country FE & & $\checkmark$ & & $\checkmark$ \\
\hline Observations & 874 & 874 & 874 & 874 \\
$R^{2}$ & 0.230 & 0.691 & 0.223 & 0.938 \\
\hline
\end{tabular}

${ }^{*} p<0.10,{ }^{* *} p<0.05,{ }^{* * *} p<0.01$

Notes: t-statistics are reported in parentheses. The standard errors for every regression are clustered at the website-country level. We aggregate every dependent variable to the website-country-product type-week level between weeks 4 and 14, including both weeks 4 and 14 (January 9th - April 5th). The dependent variable in the regression reported in the first and second column is AUC. The dependent variables in the third and fourth column is the MSE. The regression results reported in column (1) and (3) do not include website or website-country fixed effects, whereas those reported in column (2) and (4) include these fixed effects. 
Table 19: Prediction Error and Scale of Data

\begin{tabular}{lcccc}
\hline & $(1)$ & $(2)$ & $(3)$ & $(4)$ \\
& AUC & AUC & MSE & MSE \\
\hline $\log$ (Recorded Searches) & $0.0154^{*}$ & 0.0178 & -0.00435 & 0.000937 \\
Constant & $(1.84)$ & $(0.98)$ & $(-0.88)$ & $(0.15)$ \\
& $0.505^{* * *}$ & $0.510^{* *}$ & $0.191^{* * *}$ & 0.0987 \\
Product Category Controls & $(4.60)$ & $(2.45)$ & $(2.82)$ & $(1.31)$ \\
Week FE & $\checkmark$ & $\checkmark$ & $\checkmark$ & $\checkmark$ \\
Country FE & $\checkmark$ & $\checkmark$ & $\checkmark$ & $\checkmark$ \\
Website $\times$ Country FE & $\checkmark$ & $\checkmark$ & $\checkmark$ & $\checkmark$ \\
\hline Observations & & $\checkmark$ & & $\checkmark$ \\
$R^{2}$ & 874 & 874 & 874 & 874 \\
\hline$*$
\end{tabular}

${ }^{*} p<0.10,{ }^{* *} p<0.05,{ }^{* * *} p<0.01$

Notes: t-statistics are reported in parentheses. The standard errors for every regression are clustered at the website-country level. We aggregate every dependent variable to the website-country-product type-week level between weeks 4 and 14, including both weeks 4 and 14 (January 9th - April 5th). The dependent variable in the regression reported in the first and second column is AUC. The dependent variables in the third and fourth column is the MSE. The regression results reported in column (1) and (3) do not include website or website-country fixed effects, whereas those reported in column (2) and (4) include these fixed effects.

Table 20: Sliding Window Data Scale and Aggregate Prediction Error

\begin{tabular}{lcccc}
\hline & $(1)$ & $(2)$ & $(3)$ & $(4)$ \\
& AUC & AUC & MSE & MSE \\
\hline $\log$ (Two Week Search Total) & $0.0158^{*}$ & & -0.00439 & \\
& $(1.88)$ & & $(-0.87)$ & \\
$\log$ (Three Week Search Total) & & $0.0161^{*}$ & & -0.00440 \\
& & $(1.92)$ & & $(-0.86)$ \\
Constant & $0.651^{* * *}$ & $0.479^{* * *}$ & 0.0942 & $0.192^{* *}$ \\
& $(5.34)$ & $(4.05)$ & $(1.28)$ & $(2.56)$ \\
Product Category Controls & $\checkmark$ & $\checkmark$ & $\checkmark$ & $\checkmark$ \\
Week FE & $\checkmark$ & $\checkmark$ & $\checkmark$ & $\checkmark$ \\
Country FE & $\checkmark$ & $\checkmark$ & $\checkmark$ & $\checkmark$ \\
Website $\times$ Country FE & & $\checkmark$ & & $\checkmark$ \\
\hline Observations & 868 & 861 & 868 & 861 \\
$R^{2}$ & 0.129 & 0.129 & 0.140 & 0.142 \\
\hline$* p<0.10{ }^{* *} p<0.05, * * *$ \\
& & & &
\end{tabular}

${ }^{*} p<0.10,{ }^{* *} p<0.05,{ }^{* * *} p<0.01$

Notes: t-statistics are reported in parentheses. The standard errors for every regression are clustered at the website-country level. We aggregate every dependent variable to the website-country-product type-week level between weeks 4 and 14, including both weeks 4 and 14 (January 9th - April 5th). The dependent variable in the regression reported in the first and second column is AUC. The dependent variables in the third and fourth column is the MSE. The regression results reported in column (1) and (3) do not include website or website-country fixed effects, whereas those reported in column (2) and (4) include these fixed effects. The Two Week Search Total and Three Week Search Total variables are computed by summing the total number of searches observed for each observation over a sliding window of two weeks and three weeks, respectively. 